\title{
The Search for Intergalactic Hydrogen Clouds in Voids
}

\author{
Curtis V. Manning \\ Astronomy Department, University of California, Berkeley, CA 94720 \\ cmanning@astro.berkeley.edu
}

\begin{abstract}
I present the results of a search for intergalactic hydrogen clouds in voids. Clouds are detected by their HI Ly $\alpha$ absorption lines in the HST spectra of low-redshift AGN. The parameter with which the environments of clouds are characterized is the tidal field, for this places a lower limit on the cloud mass-density which is dynamically stable against disruption. The summing of the tidal fields along these lines of sight is managed by the use of galaxy redshift catalogs. The analytical methodology employed here has been designed to detect gas clouds whose expansion following reionization is restrained by dark matter perturbations. The end-products of the analysis of data are the cloud equivalent width distribution functions (EWDF) of catalogs formed by sorting clouds according to various tidal field upper, or lower limits.

Cloud EWDFs are steep in voids $(d \log (d \mathcal{N} / d z) / d \log (\mathcal{W})=\mathcal{S} \sim-1.5 \pm 0.2)$, but flatter in high tidal field zones $(\mathcal{S} \sim-0.5 \pm 0.1)$. Most probable cloud Doppler parameters are $\sim 30 \mathrm{~km} \mathrm{~s}^{-1}$ in voids and $\sim 60 \mathrm{~km} \mathrm{~s}^{-1}$ in proximity to galaxies. In voids, the cumulative line density at low $\mathrm{EW}(\mathcal{W} \simeq 15 \mathrm{~m} \AA)$ is essentially equal to that of the mean EWDF, $\sim 500$ per unit redshift. The void filling factor is found to be $0.87 \leq f_{v} \leq 0.94$. The void EWDF is remarkably uniform over this volume with a possible tendency for more massive clouds to be in void centers. The size and nature of the void cloud population suggested by this study is completely unanticipated by the results of published 3-D simulations, which predict that most clouds are in filamentary structures around galaxy concentrations, and that very few observable absorbers would lie in voids. Strategies for modeling this population are briefly discussed.
\end{abstract}

Subject headings: intergalactic medium — quasars:absorption lines - dark matter - galaxies:halos

\section{Introduction}

Suppose we divide the universe into two complementary parts; one galaxy-dominated, and the other what might be called space-dominated, or void. While we know the primary constituents of galaxy dominated space (hereafter, GDS), little is known of voids. What lies in voids? The presence of Ly $\alpha$ absorbers in lowredshift spectra raises the possibility that some may be in voids. What is the nature of Ly $\alpha$ absorbing clouds, and how does their nature change as one looks from void to GDS? What might be the long-term physical interaction between voids and the GDS? What remnants of primordial perturbations remain in voids to this day? What is the indicative total mass density in voids? How does this compare to predictions based on numerical simulations? These are questions which motivated the present investigation. Let us take a look at the clouds of the local universe; let us see what they are doing, with particular attention to those which are isolated. 


\subsection{Clouds Observed}

To begin the study of the effect of environment on the nature of intergalactic hydrogen clouds one needs the equivalent width (EW) of their absorption at the Ly $\alpha$ resonance $\left(\lambda_{\alpha}=1215.67 \AA\right)$, and their line profiles, characterized by the Doppler parameter $b\left(\mathrm{~km} \mathrm{~s}^{-1}\right)$, needed to calculate the column density of HI from the equivalent width. In addition, we shall require a parameter that will discriminate between void, and GDS clouds (to be discussed in $\S 2$ ).

The cloud population is characteristically measured by its line density $d \mathcal{N} / d z$, where $\mathcal{N}$ is the number of clouds with column density greater than some $N_{H I}$. The line density may vary with redshift. This evolution has been expressed as a power of $(1+z) ; d \mathcal{N} / d z=(d \mathcal{N} / d z)_{0}(1+z)^{\gamma}$ (Sargent et al. 1980, hereafter SYBT), where $\gamma$ is sometimes known as the evolution parameter. Before the Hubble Space Telescope (HST) made observation of low-redshift Ly $\alpha$ clouds possible, our ideas about the local population of absorbers were based on extrapolations from the observations of highly redshifted absorption spectra. These studies showed a strong evolution of the line density $d \mathcal{N} / d z$ with time ${ }^{1}$.

A series of papers from the HST Quasar Absorption Line Key Project provided information on clouds at redshifts $z \lesssim 1.5$ using the Faint Object Spectrograph (FOS) (Bahcall et al. 1991, 1992, 1993, 1996; Weymann et al. 1998). These papers provided palpable proof that the evolution rate of Ly $\alpha$ clouds at low redshift is substantially lower than at higher redshift. Weymann et al. (1998) show that for clouds with no associated metal-line absorbers, the data is consistent with $\gamma=0$ at $z<1.5$. For HI clouds with associated metal lines, $\gamma$ is in the range of 1.24 to 1.55 , depending on sample selection criteria. These absorbers have been interpreted as arising from galactic halos (SYBT).

Weymann et al. (1998) demonstrated that at low redshifts $\gamma$ declines with EW (see their Fig. 7) indicating that low-EW clouds are positively evolving (i.e., becoming more numerous) relative to larger absorbers. HI-only clouds with large EW ( $2400 \mathrm{~m} \AA$ ) were found to have evolution parameters $\gamma \simeq 0.6$, while the smaller clouds with $\mathcal{W}=350$ to $100 \mathrm{~m} \AA$ have $\gamma \sim 0.5$ to -0.5 , respectively. The evolution parameter of very low EW clouds at low redshift is still unknown, but the large redshift frequency of low EW absorbers found in Penton, Stocke, \& Shull 2000 (hereafter PSSI) suggests that the correlation between $\gamma$ and EW may continue as the EW approaches $10 \mathrm{~m} \AA$.

The HST has brought the study of Ly $\alpha$ absorbers down to a redshift at which cloud environments might be studied. An early study of the sightline to 3C 273 (Morris et al. 1993) pointed out the tendency of clouds to avoid clusters of galaxies, and commented on the existence of one large but very isolated cloud 2. Subsequently, other groups (Lanzetta et al. 1995, Tripp, Lu, \& Savage 1998, Chen, Lanzetta, Webb, \& Barcons 1998) investigated the proximity of clouds to galaxies, finding an anticorrelation between cloud equivalent width and the projected radius to the nearest galaxy. The same relationship was found to hold out to $z \approx 0.8$ (Chen et al. 1998, 2001). Tripp et al. (1998) found that the anticorrelation may extend to projected radii of $\sim 2 \mathrm{Mpc}$ from the nearest galaxy, suggesting that galaxies may affect cloud distributions at very large galactocentric distances. Tripp et al. (1998) claimed that clouds beyond this radius had a distribution of equivalent widths similar to those clustered around galaxies, but that "void" clouds appear to be generally smaller. While galactic impact parameters within $\sim 600 \mathrm{kpc}$ were found to reliably produce

\footnotetext{
${ }^{1} \mathrm{Lu}$ et al. (1991); Kim et al. (1997) show $\gamma \simeq 2.75 \pm 0.29$ and $2.78 \pm 0.71$ respectively, though Bechtold (1994) found a slower evolution $\gamma \simeq 1.89 \pm 0.28$.

${ }^{2}$ The cloud in question is the highest wavelength cloud (point with error-bar) in Fig. 6a, in the sightline to 3c273, with an equivalent width of $297 \mathrm{~m} \AA$
} 
absorption systems, outside this radius, increasingly frequent 'misses' were recorded, implying a declining covering factor with increasing radius at a given sensitivity limit.

The view that these clouds are physically associated with galaxies is challenged by Davé et al. 1999 . They claim that the absorption systems are associated with uniformly dense filamentary structures seen in 3-D hydrodynamical simulations, rather than self-gravitating clouds physically associated with galaxies and groups of galaxies. Whether associated with galaxies or filaments, there does appear to be a correlation showing the effects of proximity to galaxies. The large physical extent of the radial trend in EWs noted by Tripp et al. (1998), and the impression of an increasing cloud number density toward galaxies, suggests that the surrounding void edges are the source of clouds which are falling into the gravitational potential wells of galaxies and groups of galaxies. A very similar distribution of clouds is detected out to redshifts $z \simeq 0.89$ (Chen et al. 1998, 2001), suggesting that such infall is a long-term feature of the universe. Other lines of evidence favoring a substantial accretion rate of centrally condensed clouds are to be found in the roughly steady, or increasing star formation rate in the Galaxy disk (Maciel \& Rocha-Pinto 1998), the large dispersion of metallicities of disk stars as a function of the epoch of their formation (Edvardsson et al. 1993; Carraro et al. 1998), photometric asymmetries and other signs of accretion of low-metallicity gas in field spirals (Zaritsky 1995; Zaritsky \& Rix 1997; Haynes 2001), the predominance of negative barocentric velocities for high velocity clouds (HVC) in the Local group (Blitz et al. 1999; Wakker et al. 1999), and the existence of a "compact" subclass of infalling $\left(\sim 100\right.$ barocentric $\left.\mathrm{km} \mathrm{s}^{-1}\right)$ HVCs with cold, compact cores and ionized halos (Braun \& Burton 2000).

\subsection{Hydro Simulations}

The current view of the nature of the structure and content of the universe at low redshift is strongly affected by results of numerical cosmological simulations. Various 3-dimensional simulations have been used to model the higher redshift Ly $\alpha$ cloud population (e.g., Hernquist et al. 1996; Mücket et al. 1996; Davé et al. 1999; Cen \& Ostriker 1999), and some of them have been extended to low redshift (Riediger, Petitjean, \& Mucket 1998; Theuns, Leonard, \& Efstathiou 1998; Davé et al. 1999). These simulations suggest that the clouds with columns $\log N_{H I} \lesssim 14$ are produced in the filaments of roughly homogeneous gas, some of which may still be in partial participation with the Hubble flow (Riediger et al. 1998; Theuns et al. 1998; Davé et al. 1999), whereas the higher column density clouds are found in close proximity to galaxy halos.

Very few of the simulations concern themselves with voids per se, so some effort must be taken to extract predictions. Riediger et al. (1998) found it helpful to separate clouds into two groups - shocked $P_{s}$ and unshocked $P_{u}$. Shocked clouds result from convergent gas flows. It is thought that the unshocked clouds lie outside of the filamentary structures, and are expanding with the Hubble flow so that their column densities would decline steeply with declining redshift $\left(N_{H I} \propto(1+z)^{5}\right)$ at higher redshifts where $J(z)$ is fairly constant (Riediger et al. 1998; Davé et al. 1999), so that this component produces negligible absorption for $z \leq 3.0$. Cen \& Ostriker (1999) argue that from $1 / 2$ to $2 / 3$ of all baryons are in the filamentary structures constituting a warm to hot medium $\left(10^{5} \mathrm{~K}\right.$ to $\left.10^{7} \mathrm{~K}\right)$ with a volume filling factor $\sim 0.1$. This is the population referred to by Riediger et al. (1998) as "shocked", and by Davé et al. (1999) as producing columns $\log N_{H I} \lesssim 14\left(\mathrm{~cm}^{-2}\right)$ at low redshift. Void clouds are not expected to produce any significant Ly $\alpha$ absorption (Davé, priv. comm. 2001). However, a simulation by Cen \& Ostriker (1999) finds that about $26 \%$ of baryons are located in a "warm" medium $\left(T<10^{5} \mathrm{~K}\right)$ with a filling factor of 0.9 - this would be the "unshocked" population $P_{u}$ whose contribution to the line density faded rapidly at redshifts less than 3. Thus, these simulations suggest that the unshocked region is what we would call "void", and has a current 
density of $\bar{\rho}_{U} \approx 0.26$ of the average, and that filaments, with filling factor 0.1 , have a density $\bar{\rho}_{S} \gtrsim 7$ times the average, so that the void matter density would be less than $4 \%$ of that in the filaments. The issue of the void matter density is addressed directly by Einasto et al. (1994) through simulations of CDM cosmogony, showing that matter flows out of low density environments and into high density areas, resulting in an estimated $15 \%$ of matter being located in present-day voids. El-Ad \& Piran (1997) estimate a void matter density of $10 \%$ of the mean based on observation of the galaxy content of nearby voids.

Given the above, it seems fair to conclude that the predictions of simulations are for isolated (i.e., void) clouds to be evolving rapidly, and for the line density in voids, at EWs detectable with current technonogy, to be quite low. The overwhelming majority of absorption systems are expected to be produced by gas associated with the filamentary structures having warm to hot temperatures and a filament filling factor $f_{f} \sim 0.1$ (Davé et al. 1999).

\subsection{Structure Formation}

The conclusions from more theoretically-based studies of structure formation are somewhat different. In semi-analytic simulations of very high redshifts (Abel et al. 1998; Bromm, Coppi, \& Larson 1999; Nakamura \& Umemura 2001), molecular hydrogen, formed shortly after recombination, provides cooling for low-mass perturbations which results in the formation of Population III stars at $z \simeq 20$ in halos with halo velocities as low at $\sim 1.5 \mathrm{~km} \mathrm{~s}^{-1}$ suggesting that even very small halos with masses in the range $10^{5}$ to $10^{9} \mathrm{M}_{\odot}$, would have formed stars. However, this field currently suffers from a "missing halos" problem; there should be many more dwarf galaxies within the Galaxy's halo under the favored cold dark matter cosmogony (CDM) than are in fact observed. Estimates of the magnitude of the discrepancy between predicted and observed low-mass galaxies range from a few (Kauffmann et al. 1993) to a factor of $\sim 50$ (Klypin et al. 1999; Moore et al. 1999a). It appears that the discrepancy manifests itself increasingly below halo velocities of $\sim 40 \mathrm{~km} \mathrm{~s}^{-1}$. The deficit in numbers between predicted low-mass mini-galaxies, and that suggested by observations of Local Group galaxies implies that the overwhelming majority of small halos $\left(v_{c} \lesssim 25 \mathrm{~km} \mathrm{~s}^{-1}\right)$, for whatever reason, did not in fact generally form stars. Therefore, these halos could very well be present in the universe today as clouds. But while as mini-galaxies they could survive the tidal fields around large galaxies, as clouds the ram pressure of dissipated gas around galaxies and groups of galaxies would cause the stripping of halos of their baryons, rendering them invisible. Therefore, the cloud density may be less than the numbers of mini-galaxies predicted to have survived to the present with identical halo velocities due to their disruption in dense environments. Their remnants may remain as isolated (Ly $\alpha$ clouds), or they may be currently falling into galaxy halos and identified as HVCs as suggested by Klypin et al. (1999) and Grebel, Braun, \& Burton 2000. Yet it is claimed (Klypin et al. 1999) that their numbers are not inconsistent with the full deficit of halos.

\subsection{This Paper}

The general success of bottom-up galaxy formation scenarios in predicting large-scale structure provides considerable justification for accepting the notion that halos of sub-galactic size were already in place at the epoch of reionization with their baryons somewhat relaxed into their halos. Yet the paucity of mini-galaxies today suggests that cooling could not generally have proceeded to the point of star formation, and that somewhat larger clouds, formed later but with the greater cooling rates afforded by their stronger self- 
gravity, formed the first stars.

It is reasonable to suppose that following reionization, baryons were heated and flowed outward (Barkana \& Loeb 1999), but that they may still be in the proximity of those halos. The dark halos of clouds provide a means to restrain the dispersal of baryons after the epoch of reionization. Though the ability to retain the outflow is dependent on the halo model, this could help to explain the apparent endurance of low equivalent width clouds, as suggested by the low observed values of their evolution parameter (Weymann et al. 1998).

High-sensitivity observations of low-redshift Ly $\alpha$ clouds may provide a means to corroborate these assertions. PSSI used HST/GHRS/G160M to study the absorption spectra of bright, low redshift Seyfert and BL Lac galaxies at high spectral resolution $\left(\sim 19 \mathrm{~km} \mathrm{~s}^{-1}\right.$, or $\left.80 \mathrm{~m} \AA\right)$. Their observations were supplemented by archival data from the same instrument. Some of these spectra reached a "sensitivity" (see §4.2) as low as about $12 \mathrm{~mA}$. One of the important outcomes of their analysis (Penton, Shull, \& Stocke 2000a, hereafter PSSII) was the determination of the mean HI equivalent width distribution function $f(\mathcal{W})$. Results showed that the redshift frequency of Ly $\alpha$ clouds with $\mathcal{W} \gtrsim 12 \mathrm{~mA}$ (HI columns $N_{H I} \gtrsim 10^{12.3} \mathrm{~cm}^{-2}$ for $b \simeq 25 \mathrm{~km} \mathrm{~s}^{-1}$ ) approach $d \mathcal{N} / d z \simeq 450$ per unit redshift. In their modeling of the clouds, PSSII suggested that $20-45 \%$ of all baryons in the low redshift universe are associated with these clouds.

In contrast to expectations based on the simulations mentioned above, both PSSI and Tripp et al. (1998) show that significant numbers of clouds (up to 50\%) appear to be very isolated. One wonders, what exactly is going on in galaxy voids? This paper is an attempt to answer that question. The paper begins with an analysis of the observational basis for various measurable cloud quantities ( $\$ 2)$, seeks the proper way to specify the environment of a cloud, and then shows how galaxy redshift catalogs can be used to measure it $(\S 3)$. The source and nature of the data used in this study are presented in $\S 4$. Details on the method by which mass is attributed to galaxies, and how tidal fields are summed, are presented in $\S 5$. Section 6 presents the distribution functions of clouds specified by tidal field upper and lower limits. In addition, a variational approach is used to investigate the effects of various assumptions on the output distribution functions. There is a discussion ( $(7)$ and conclusions $(\S 8)$.

Though the effect of employing different cosmologies on the results of this analysis is minimal, a cosmology must be specified, as future modeling will require a cosmological context. A flat FRW cosmology with $\Lambda=0.7$, and $h=0.75$ is used, unless otherwise noted. The cosmic baryon density is assumed to be $\Omega_{b} / \Omega_{m}=0.11$.

\section{About Clouds}

Before studying these clouds in particular, it may be helpful to review their basic observational relationships. The cumulative line density $d \mathcal{N} / d z$ of evenly distributed clouds of comoving number density $n$ and which produce a column density of $\geq N_{H I}$ (or, alternatively, an EW $\geq \mathcal{W}$ ) at projected distance $r_{p}$ from the center of a spherical cloud is,

$$
\frac{d \mathcal{N}}{d z}=n \pi r_{p}^{2}(1+z)^{3} \frac{d l}{d z},
$$

where $d l$ is the element of proper path length, such that the comoving distance coordinate element $d r=$ $(1+z) d l$, and the column density is formally defined by the integral of the HI density over a column of length $L$ through the cloud at a projected radius $r_{p}$,

$$
N_{H I}=\int_{L} n_{H I}\left(r \geq r_{p}\right) d L .
$$


We also have, by way of a definition of $\gamma$,

$$
\frac{d \mathcal{N}}{d z}=\left(\frac{d N}{d z}\right)_{0}(1+z)^{\gamma}
$$

Solved for $n$, Eqs. 1 and 3 yield,

$$
n=\left(\frac{d \mathcal{N}}{d z}\right)_{0} \frac{(1+z)^{\gamma-3}}{\pi r_{p}^{2}} \frac{d z}{d l}
$$

For a flat universe with non-zero cosmological constant,

$$
\frac{d l}{d z}=\frac{R_{0}}{(1+z) \sqrt{\left.\Omega_{m}(1+z)^{3}+\Omega_{\Lambda}\right)}},
$$

(Scott D. and Silk) where $R_{0}=c / H_{0}$. Assume that the radius at which a given column density occurs in an individual cloud is

$$
r_{p} \propto(1+z)^{\epsilon},
$$

then taking the derivative of Eq. 4 with respect to $z$ we find,

$$
\frac{1}{n} \frac{d n}{d z}=\frac{\gamma+2 \epsilon-3}{(1+z)}-\frac{d^{2} l / d z^{2}}{d l / d z}
$$

The last term is,

$$
\frac{d^{2} l / d z^{2}}{d l / d z}=-\frac{1}{1+z}\left[1+\frac{3 / 2}{\left(1+\Omega_{\Lambda} /\left(\Omega_{0}(1+z)^{3}\right)\right)}\right] .
$$

Substitute the above into Eq. 6 to find,

$$
\frac{1}{n} \frac{d n}{d z}=\frac{1}{1+z}\left[\gamma+\frac{3 / 2}{1+\Omega_{\Lambda} /\left(\Omega_{0}(1+z)^{3}\right)}+2 \epsilon-2\right] .
$$

Assume that the comoving number density of some population of clouds is constant so that $1 / n(d n / d z)=0$. Then,

$$
\gamma=2-2 \epsilon-\frac{3(1+z)^{3} \Omega_{m}}{2 \sqrt{\Omega_{m}(1+z)^{3}+\Omega_{\Lambda}}} .
$$

Let us consider the specific case in which there are associated metal lines. It is probable that the radius at which metal lines are detected is fairly constant if they require a hot halo to maintain them in ionized form. In that case, the cross section for absorption is constant, and $\epsilon=0$. With $d n / d z=0$ at $z=0$, we find the evolution parameter for metal-line clouds is

$$
\gamma_{\text {metal }}=1.55
$$

for the adopted cosmological parameters $(\S 1)$, consistent with the evolution parameter for metal-line HI clouds observed byWeymann et al. (1998). However, when we are dealing with primordial Ly $\alpha$ clouds that are DM-held, the radius at which a given column density is observed may change. We decompose $\epsilon$ into the effects of a changing metagalactic flux and the cloud density profile. Assume that

$$
\begin{aligned}
& J(z)=J_{0}(1+z)^{\xi} \\
& \rho(r)=\rho_{0} r^{-\delta} .
\end{aligned}
$$

Equation 10 entails a temporally static matter distribution for each cloud. This may be unrealistic; small clouds may still be expanding, and larger clouds may be recollapsing. However, analytical modeling at this 
level of detail is clearly inferior to hydrodynamic simulations, so we forgo this in favor of the simplification that clouds are static. Since optically thin gas has an HI fraction inversely proportional to $J_{0}(z)$, and directly proportional to $n_{H}^{2}$, then $n_{H I} \propto r^{-2 \delta} /(1+z)^{\epsilon}$. The column density at a cloud projected radius $r_{p}$ is then $N_{H I} \propto r_{p}^{-(2 \delta-1)} /(1+z)^{\epsilon}$. The impact parameter $r_{p}$ at which the cloud has a neutral column $N_{H I}$ varies as,

$$
r_{p} \propto(1+z)^{\xi /(2 \delta-1)} .
$$

Apparently we may substitute $\xi /(2 \delta-1)$ for $\epsilon$ in Eq. 7 and 8 . If $\xi \sim 3$ or so, as suggested by the work of Shull et al. (1999), and $\delta \sim 1.75$, a somewhat flattened baryon distribution, then $\epsilon=1.2$, and at $z=0$, Eq. 7 yields,

$$
\gamma_{c l} \simeq-0.85
$$

If we can extend the $\gamma-E W$ correlation presented in Weymann et al. (1998) down to, say, $12 \mathrm{~m} \AA$, then the above is in agreement.

But what are the predictions of simulations? As Riediger et al. (1998) point out, if the ionizing flux is approximately constant, then the column density for clouds expanding with the Hubble flow is $N_{H I} \propto$ $n_{H}^{2} L \propto(1+z)^{5}$. Therefore, if $J_{0} \propto(1+z)^{\xi}$, where $\xi \simeq 3.0$, then $N_{H I} \propto(1+z)^{2}$.

To summarize, the analytical approximations of Davé et al. (1999) and Riediger et al. (1998) are based on the presumption of homogeneous clouds of negligible mass which expand freely but have sharp edges, whereas the view presented in this paper contends that the clouds are self-gravitating, and have the gas density gradients characteristic of self-gravitating clouds. As suggested by Davé et al. (1999), their cloud model can explain the observed low $\gamma$ for low EW clouds by citing the effects of a decline in flux of the ionizing background with time, but to accomplish this $\xi$ (Eq. 9) must be of order 4.5, rather more than suggested by the work of Shull et al. (1999). Our preference is that cloud density gradients in conjunction with a decline in $J_{0}(z)$ are responsible for the low observed cloud evolution parameter (Weymann et al. 1998).

\section{The Environments of Clouds}

Self-gravitating clouds are delicate structures and can be easily disrupted. We are searching for one characteristic which by its absence allows clouds to abide, while in its presence the cloud is destroyed. Intuitively, isolation corresponds to the lack of that factor which destroys clouds. Hence, our parameter deals with the proximity to galaxies and groups of galaxies. Heretofore, the degree of isolation of clouds has been generally characterized by the distance (in projected radius and velocity) to the nearest galaxy. However, one megaparsec from a small galaxy is not the same as one megaparsec from a giant. Optimally, one should weight the influences of galaxies by their mass and distance. Besides the effects of ionizing radiation, which is here assumed to be uniform over the small range of redshifts of the data to be studied, the principal constraints on the physical integrity of clouds is from shear forces or ram pressure, and the strength of the tidal field. In addition, the heightened probability that a small halo would be accreted to a larger one is a direct function of ambient density; it is expected to produce an effect similar to that of a tidal field - a flattening of the cloud EW distributions in high density areas. For isolated clouds, only tidal fields are relevant, though the flux of ionizing photons may cause heating which will help to disperse the baryonic content of small clouds. Because a uniform distribution of mass will have no tidal effect on a cloud, galaxies are the main sources of tidal fields on large scales. 


\subsection{Tides produced by galaxies}

If one takes the second spatial derivative of the summed gravitational potentials of galaxies at a particular point, one gets a quantity indistinguishable from the tidal field. One can imagine, then, that minima in the magnitude of tidal fields would occur in galaxy voids. The tidal field $T$ is in fact a function expressible in units of inverse time squared, so that if it is multiplied by the Hubble time squared it becomes a dimensionless quantity $\mathcal{T}=T / H_{0}^{2}$. The dimensionless form $\mathcal{T}$ is hereafter referred to as the "tidal field" parameter. Tides may be of vector or scalar form. In this paper, a scalar tide is calculated, though we must first consider its vector form. For an individual galaxy, the only non-zero component of $\mathcal{T}$ is radial;

$$
\mathcal{T}_{R}=-\frac{1}{H_{0}^{2}} \frac{d}{d R}\left(\frac{G M(R)}{R^{2}}\right)
$$

In summing the effects of many galaxies at a given point along the line of sight (LOS), we must decompose this into Cartesian components. For instance, the $x$-component, transverse to the LOS, is

$$
\mathcal{T}_{x}=\frac{2 G M(R)}{H_{0}^{2} R^{4}}|\Delta x|
$$

where $R=\sqrt{\Delta x^{2}+\Delta y^{2}+\Delta z^{2}}$. The components transverse to the observer's LOS are calculated using the angular diameter distance pertinent to the cosmological model employed in this paper. The proper displacement $\Delta z$ is found using Eq. 5 ( $(2)$. That is, the radial (LOS) position is determined by the redshift alone; no allowance is made for peculiar velocities. This causes some positional error, which will be discussed in $§ 5.5$. In this manner, the contributions of each galaxy can be added, then combined in quadrature to arrive at an averaged scalar tidal field $\mathcal{T}$ acting on a cloud,

$$
\mathcal{T}=\frac{\sqrt{\mathcal{T}_{x}^{2}+\mathcal{T}_{y}^{2}+\mathcal{T}_{z}^{2}}}{3}
$$

Let us see how the tidal field works to disrupt clouds. The condition for stability to tidal disruption is as follows,

$$
H_{0}^{2} \mathcal{T} r \leq \frac{G m(r)}{r^{2}}
$$

where $m(r)$ is cloud mass summed to radius $r$. When a cloud is close to a single galaxy, then the radial form will be more pertinent. By application of Eq. 12 to single galaxy, the tidal field is,

$$
\mathcal{T}_{R}=\frac{G M(R)}{H_{0}^{2} R^{3}}\left(2-\frac{d \log M}{d \log R}\right) .
$$

For an isothermal halo, $d \log M / d \log R$ is unity when $R \leq R_{t}$, and zero beyond that. The standard halo model is that of Navarro, Frenk, \& White 1996, 1997 (hereafter NFW), which we discuss in more detail below (\$5.1.2). The above derivative for the NFW halo does not approximate a constant function of $r$ unless baryons "conspire" to produce a flat rotation curve inside $r_{\max }$. However, beyond $r_{\max }$ the density profile tends toward an index of -3 , and a simple solution for $\mathcal{T}_{R}$ no longer maintains.

The simplest halo is isothermal, possibly truncated at some characteristic radius $R_{t}$. The mass of such a halo increases linearly with radius, so that one may describe this radial mass distribution by a constant, $\mathcal{K} \equiv M(R) / R$. The circular velocity of such a system is $v_{c}=\sqrt{G \mathcal{K}}$, and the total mass is $M_{\text {tot }}=\mathcal{K} R_{t}$. For a truncated isothermal halo, Eq. 13 yields,

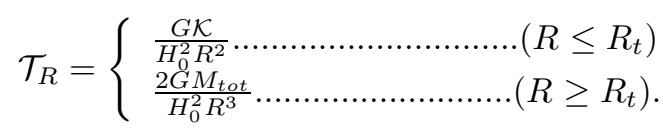


Note that the effect of halo mass truncation on the tidal field is to produce a discontinuity at $R_{t}$. While a sharp truncation is not realistic, it will not produce a significant effect on the distributions of clouds as we shall in all cases assume a total mass.

In the context of a specific cloud, Eq. 15 can be written,

$$
\frac{H_{0}^{2} \mathcal{T}}{G} \leq \frac{m}{r^{3}}=\frac{4 \pi}{3} \bar{\rho}(r)
$$

where $\bar{\rho}(r)$ is the average over-density of the cloud within radius $r$. Thus, the requirement for stability can be stated,

$$
\bar{\rho} \geq \frac{3 H_{0}^{2} \mathcal{T}}{4 \pi G}=2 \mathcal{T} \rho_{\text {crit }},
$$

where $\rho_{\text {crit }}$ is the critical density, $\rho_{\text {crit }}=3 H_{0}^{2} / 8 \pi G$. There is one important caveat to this: the proper number density of galaxies declines with decreasing redshift due to the expansion of the universe. Therefore, since the average distance of galaxies from an average void cloud is increasing with time, it is clear that in the past the tidal field on a void cloud varies roughly as $\mathcal{T} \propto(1+z)^{3}$. However, clouds which are in some proximity to a galaxy may have a weaker $\mathcal{T}$ with lookback time if the peculiar velocity toward the galaxy is greater than the $H R$. When we think of the tidal field affecting void clouds we must antedate to some extent the current $\mathcal{T}$ to get a feel for what it has been subjected to in the past. For instance, at a lookback time of $7.2 \operatorname{Gyr}(z=1)$, the tidal field affecting a void cloud will be $\sim 8$ times the present value.

Self-gravitating clouds have negative radial density gradients. The $\bar{\rho} \geq 2 \mathcal{T} \rho_{\text {crit }}$ criterion applies differentially to the clouds within which $M(r) / V(r)>2 \mathcal{T} \rho_{\text {crit }}$. Though tidal fields affect all clouds, only the smallest are destroyed. Thus the effects of tides will be manifested over the distribution of cloud sizes, and hence over the whole range of column densities and EWs. The physical effect will be a tidal truncation, reducing the average column density a cloud would produce for a given halo velocity. It is expected to affect low-mass clouds more dramatically than more massive clouds.

A cloud near a galaxy will experience a radial tidal field, and it is unlikely to have experienced a higher tide in the past, given its probable infall into the galaxy's (or group) potential well. An example may clarify the utility of $\mathcal{T}_{R}$. A homogeneous cloud with an average over-density $\bar{\rho}=2 \rho_{\text {crit }}$ would be marginally destabilized by a tidal field of $\mathcal{T}_{R}=1.0$. A galaxy with a flat rotation curve of $v_{c}=220 \mathrm{~km} / \mathrm{s}$ would destabilize this cloud at a distance of $2.08 h_{75}^{-1} \mathrm{Mpc}$ if the galaxy halo truncates at $0.5 h_{75}^{-1} \mathrm{Mpc}$, or at 2.94 $h_{75}^{-1} \mathrm{Mpc}$ if it has an untruncated isothermal halo. Thus we would not expect to find discrete clouds of low EW in close proximity $(\lesssim 2 \mathrm{Mpc})$ to galaxies. A galaxy with an NFW halo of the same velocity would have a tidal field $\mathcal{T}=1.0$ at a distance slightly less than $2 / 3$ times that of the truncated isothermal halo.

We now turn our attention to the problem of sorting clouds by their environments. If the tidal field is calculated along the lines of sight to AGN, then if one specifies a limiting tide $\mathcal{T}$, absorption systems can be separated into two catalogs, one with $\mathcal{T}$ providing the upper limit, denoted $\mathcal{C}\left(\mathcal{T}^{U}\right)$, and the other with $\mathcal{T}$ as a lower bound, $\mathcal{C}\left(\mathcal{T}^{L}\right)$. The actual calculated value of the tidal field will depend on which galaxy halo model is used. The NFW halo is the outcome of analysis of N-body simulations, and has a "cuspy" core approaching a density $\rho \propto r^{-1}$. However, more recent, higher-resolution simulations find the core of the halo to be even more cuspy than the NFW model $\left(\rho \propto r^{-1.5}\right)$ (Moore et al. 1999b), suggesting a conflict with observed galaxies (Firmani et al. 2001; Burkert 2000; Wu \& Xue 2000). Furthermore, the NFW halo may be inconsistent with what is suggested by the steadiness of the radial dependency of the velocity dispersions of dwarf galaxies about their parent giant field galaxies to radii $r_{p} \approx 500 h_{75}^{-1} \mathrm{kpc}$ (Zaritsky \& White 1994; Zaritsky et al. 1997), far beyond the radius at which the halo has a maximum circular velocity $\left(r_{\max }\right.$ is of 
order $15-40 \mathrm{kpc}$ ). Because of these perceived problems with the NFW model, and the simplicity of the truncated isothermal halo, the latter is used as a standard model in this paper, relegating the NFW to the position of first alternative model.

It is possible to coarsely see the effect of tides in the original PSSI data by binning absorption systems according to the projected radii (with $\Delta v<500 \mathrm{~km} \mathrm{~s}^{-1}$ ) to the nearest galaxy. The results show that very low column density systems are not seen within $\sim 1 \mathrm{Mpc}$ of a galaxy (Fig. 1, top panel), but the farther one goes from the nearest galaxy (middle and bottom panels), the lower the Doppler parameters and column densities which are recorded. If void clouds were freely expanding, one would not see lower b-values with decreasing EWs, as the bulk velocity of expansion is added to the thermal broadening.

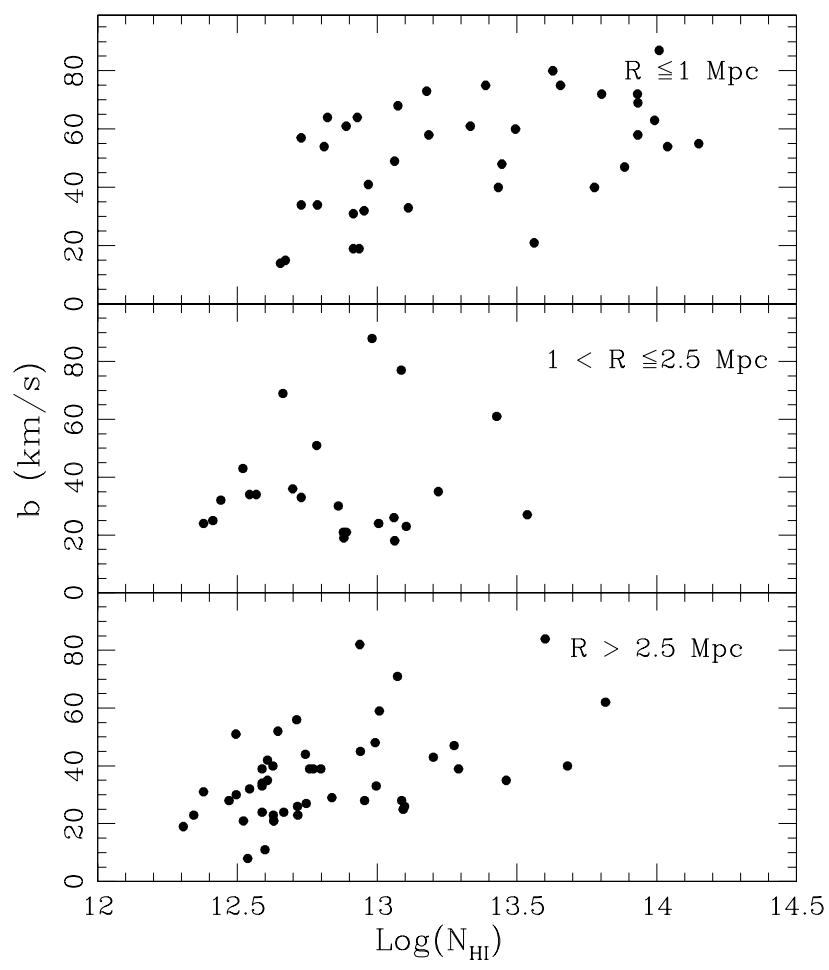

Fig. 1.- Column density as a function of b-value for data from PSSI for 3 bins of projected radius to the nearest galaxy. Radius was derived using a "retarded" Hubble flow model (clouds are placed at the galaxy redshift when they are within $\pm 300 \mathrm{~km} \mathrm{~s}^{-1}$ of the galaxy). Cloud b-values appear to have lower limits which correlate inversely with galactocentric radius. The variation of the lower limits of column densities with radius are consistent with the effects of tidal fields.

These provisional findings are consistent with the notion that tidal fields disrupt smaller clouds, and also supports the concept that the expansion of baryonic clouds are at least restrained by the gravity of their dark halos. It is reasonable to suppose that outside of massive halos, tidal fields provide the main constraint on cloud stability. We therefore formally adopt the tidal field $\mathcal{T}$ as the discriminator for cloud environments; a low $\mathcal{T}$ then indicates an environment in which cloud density is essentially unconstrained, and a high $\mathcal{T}$ would mean that clouds with density $\rho_{c l} \lesssim 2 \mathcal{T} \rho_{\text {crit }}$ would be disrupted. 


\section{The Data}

The basic data (PSSI) required for calculating the distribution $f(\mathcal{W})$ as a function of tidal field are of three types; cloud absorption-line data (wavelength $\lambda$, Equivalent width $\mathcal{W}$, Doppler parameter $b$ ), sensitivity functions, and galaxy redshift catalogs. We begin with the HST/GHRS/G160M data of PSSI along 15 sightlines toward luminous, low-redshift Seyfert and BL Lac galaxies. Table one presents 15 target RA and Dec as well as the spectral range covered by the data for each line of sight.

Table 1: Spectral targets and wavelength range

\begin{tabular}{lcccc}
\hline \hline Target & RA & Dec $(\mathrm{J} 2000)$ & $\lambda_{\min }(\AA)$ & $\lambda_{\max }(\AA)$ \\
\hline 3C 273 & $12: 29: 06.7$ & $+02: 03: 08.6$ & 1214.11 & 1301.46 \\
Arkelian 120 & $05: 16: 11.4$ & $-00: 08: 59.0$ & 1222.51 & 1258.75 \\
ESO 141-G55 & $19: 21: 14.3$ & $-58: 40: 13.0$ & 1222.51 & 1265.92 \\
Fairall 9 & $01: 23: 45.8$ & $-58: 48: 20.0$ & 1219.79 & 1276.38 \\
H1821+643 & $18: 21: 57.3$ & $+64: 20: 36.4$ & 1231.66 & 1267.75 \\
IZW 1 & $00: 53: 34.9$ & $+12: 41: 36.0$ & 1221.40 & 1257.54 \\
Mrk 279 & $13: 53: 03.4$ & $+69: 18: 29.6$ & 1222.53 & 1258.74 \\
Mrk 290 & $15: 35: 52.4$ & $+57: 54: 09.2$ & 1231.76 & 1268.99 \\
Mrk 335 & $00: 06: 19.5$ & $+20: 12: 10.5$ & 1221.41 & 1257.55 \\
Mrk 421 & $11: 04: 27.3$ & $+38: 12: 31.8$ & 1221.43 & 1257.57 \\
Mrk 501 & $16: 53: 52.2$ & $+39: 45: 36.6$ & 1221.42 & 1257.56 \\
Mrk 509 & $20: 44: 09.7$ & $-10: 43: 25.0$ & 1219.47 & 1267.84 \\
Mrk 817 & $14: 36: 22.1$ & $+58: 47: 39.4$ & 1222.57 & 1258.77 \\
PKS 2155-304 & $21: 58: 52.1$ & $-30: 13: 32.1$ & 1222.58 & 1293.67 \\
Q1230+0155 & $12: 30: 49.9$ & $+01: 15: 23.0$ & 1216.96 & 1254.23 \\
\hline
\end{tabular}

\subsection{The Absorption Systems}

Source spectra probe sightlines to low-redshift active galaxies brighter than $V \leq 14.5$ mag (Table 1). Only two of the sightlines include data beyond a redshift $z=0.045$. The well-studied sightline to $3 \mathrm{C} 273$ includes clouds with the largest maximum redshift of $z \simeq 0.07$.

The data include cloud wavelength and error, equivalent width, and its error, the resolution-corrected Doppler parameter, and its error. Some of the spectra are pre-, and some post-deployment of COSTAR (Corrective Optics Space Telescope Axial Replacement), but there is no attempt to separate them except insofar as their sensitivity functions are calculated somewhat differently. No clear trend in Doppler parameter is noticed between pre- and post-COSTAR data.

The clouds are considered to be randomly selected from a parent population - one which is effected by ambient tidal fields - within the constraints of the sensitivity of the instrument along the line of sight (LOS). The reader is referred to PSSI for a detailed description of the reduction of the HST data. Their procedure involves fitting the continuum to a polynomial by increasing the order of the polynomial until no further reductions in $\chi^{2}$ are possible. All negative spectral fluctuations from the continuum greater than 1- $\sigma$ are treated as the original absorption line list, and the lines are fitted with gaussian components accepting only those with values $12<b_{\text {obs }}<100 \mathrm{~km} \mathrm{~s}^{-1}$. The significance levels of clouds are calculated by integrating the signal to noise ratio per resolution element over the cloud (see below). 


\subsection{Sensitivity functions}

The sensitivity functions indicate what cloud EWs can be observed given the significance level imposed for the cloud catalog.

Let us begin with a review of the relationships between signal-to-noise $(S / N)$, significance level $(S L)$, EW, and sensitivity $(\mathcal{S})$. The signal $S$ of an absorption system is the integral of the counts-deficit relative to the continuum over a resolution element,

$$
S=\int_{R E}\left(f_{\text {cont }}(\lambda)-f_{\lambda}\right) S_{G}(t / 4) d \lambda \equiv \Delta n,
$$

where $S_{G}$ is the GHRS sensitivity at $\lambda$ in units counts/s/diode/ergs $/ \mathrm{s} / \mathrm{cm}^{2} / \AA$ (GHRS handbook), and the absorption system is represented by the difference of the continuum $f_{\text {cont }}$ and the observed flux $f_{\lambda}$. The time $t$ is divided by 4 because the exposures are "quarter stepped" using FP-SPLIT to help remove the effects of photocathode granularity and diode to diode detector variations. The noise $N$, based on the total counts in the continuum integrated over one $\mathrm{RE}$, is

$$
N=\sqrt{\int_{R E} f_{\text {cont }} S_{G}(t / 4) d \lambda}=\sqrt{n},
$$

so within a given RE,

$$
S / N=\frac{\Delta n}{\sqrt{n}} .
$$

Other sources of noise are said to be small so that the $\mathrm{S} / \mathrm{N}$ is within $\sim 5 \%$ of the "root n" photon statistics (Final Report, GHRS Science Verification Program, 1992). It will be assumed that "root n" statistics are accurate though it may be a small under-estimate of the true sensitivity in $\mathrm{m} \AA$. The resolution element $R E$ is estimated at 1.2 diodes for pre-COSTAR and 1.1 diodes for post-COSTAR installation (PSSI). The observed equivalent width of a cloud within a resolution element is,

$$
E W=\frac{\Delta n}{n / R E}=\frac{\Delta n}{n} R E,
$$

where $R E \simeq 80 \mathrm{~m} \AA$ (PSSI).

A cloud detected at a 4.0- $\sigma$ SL in one RE has an $\mathrm{EW} \mathcal{W}=\Delta n R E / n$ such that $\Delta n=4.0 \sqrt{n}$. The sensitivity per RE, $\mathcal{S}$, is the EW of a $4-\sigma$ cloud. Thus,

$$
\mathcal{S}=\frac{4 \sqrt{n}}{n} R E=\frac{4 R E}{\sqrt{n}}
$$

These sensitivity functions are plotted as solid lines in Fig. 2.

The total cloud EW is the sum of the EWs of each resolution element. The total significance level of the cloud is the imposed significance level times the summed equivalent width per RE divided by the sensitivity per RE. Using the equations above, terms cancel, and the SL of the cloud is,

$$
S L=\sum_{i} \frac{\Delta n(i)}{\sqrt{n(i)}} .
$$

Thus the SL of the cloud is the sum of the $\mathrm{S} / \mathrm{N}$ in each $\mathrm{RE}$ of the cloud. In practice this is done by integrating the $S / N$ over the best fit Gaussian. Thus, if a cloud of a given EW is found in one RE, and if its $E W \geq \mathcal{S}$, 
then a cloud with a similar EW, but spread over more RE's, will have the same significance level, for the counts deficit per RE $\Delta n \propto b^{-1}$, while the number of RE's spanned by the cloud is $\propto b$; in combining them the $b$-factors cancel. Thus, if a cloud appears above the sensitivity function, then it is a 4 -sigma cloud, regardless of its $b$-value.

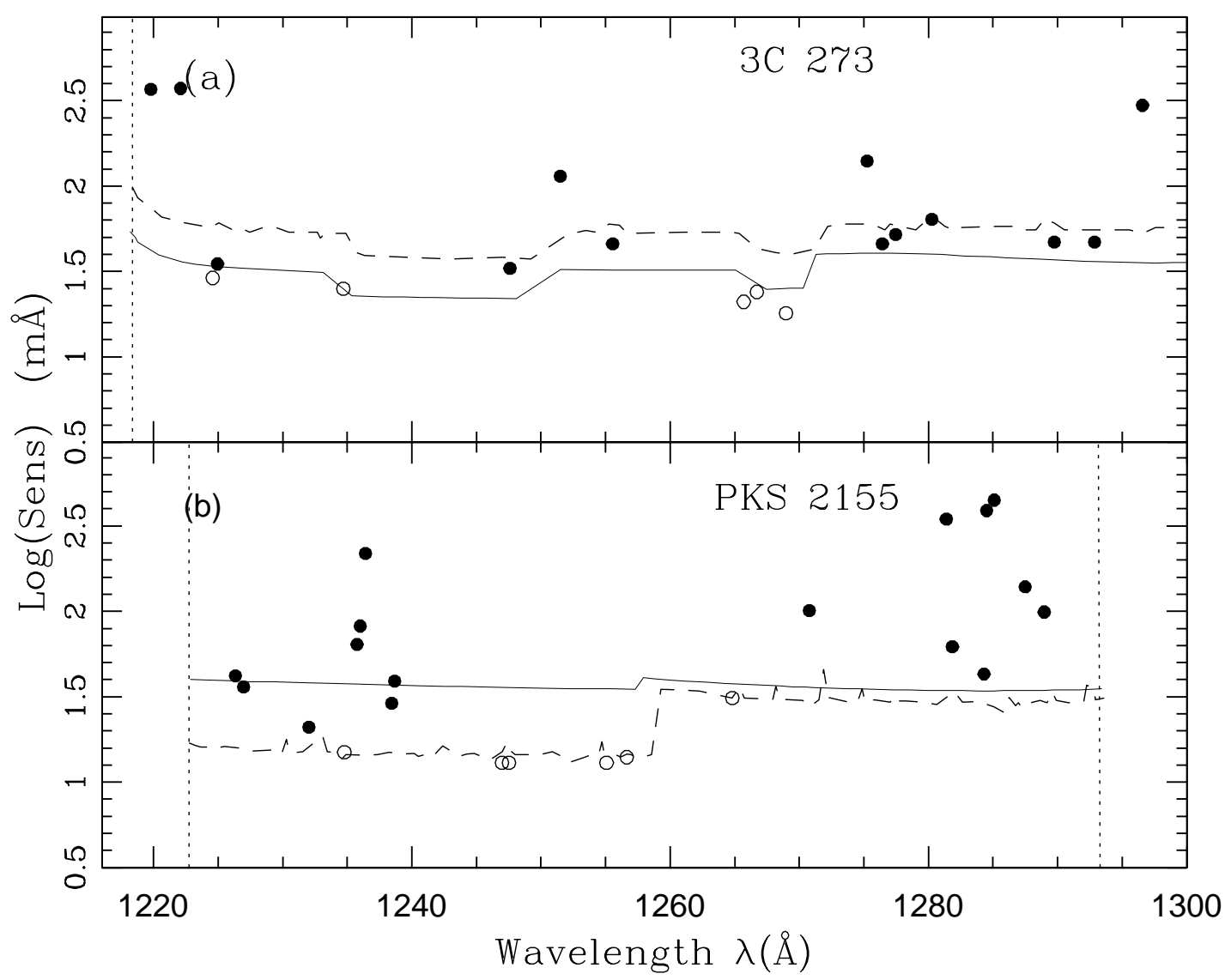

Fig. 2.- The sensitivity functions (panels a-o) as derived by the author from the flux (solid line) and extracted from PSSI on the basis of fluctuations around the continuum. The dashed lines are the sensitivity functions from PSSI. Clouds with significance greater than 4- $\sigma$ (as assigned in PSSI) are represented by solid dots; open dots are for clouds with SL in the range, $3-\sigma \leq S L \leq 4-\sigma$

The sensitivity functions published by PSSI (the dashed lines in Fig. 2) are based on the noise in the spectrum around the placed continuum. Clouds are plotted at their observed Ly $\alpha$ wavelength, and rest EW. Filled circles represent the PSSI assignment of $S L$ values $\geq 4-\sigma$, while clouds with $3-\sigma \leq S L<4-\sigma$ are shown by open symbols.

It is apparent that the two sets of sensitivity functions do not always agree. While many are in substantial agreement (e.g., Mrk 421, Mrk 279, Akn 120, Mrk 817, Q1230+0115, ESO 141-G55 and possibly Mrk 509), serious problems are apparent for IZW 1, Mrk 290, Mrk 501, Fairall 9, and H1821+643. Some sightlines 


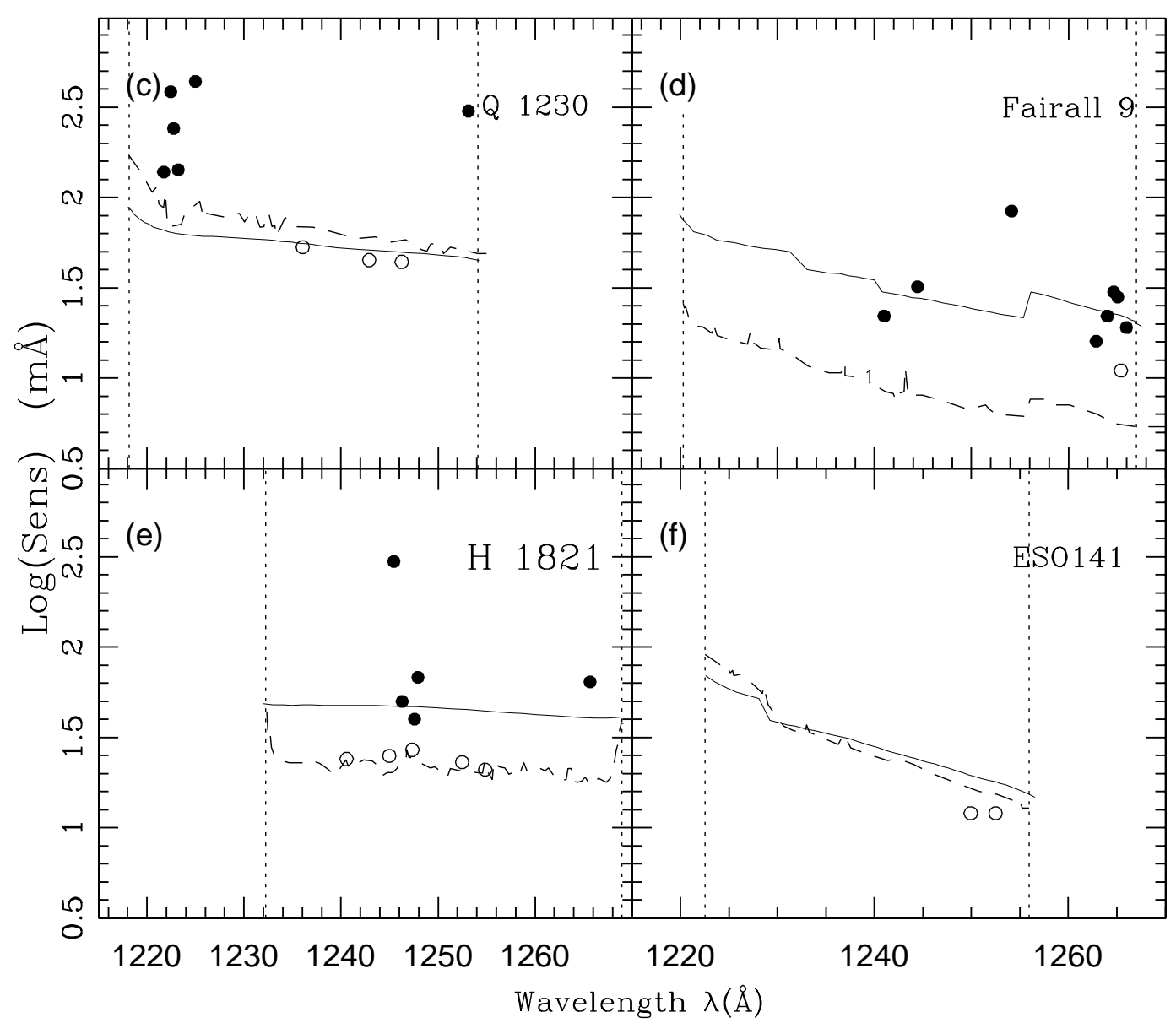

Fig. 2.- (Continued)

appear to have clouds with $S L \geq 4-\sigma$ and EWs less than the sensitivity function, or clouds with $S L<4-\sigma$ with EWs larger than the sensitivity function (e.g., Figs. 2a, d, e, j, k, and m), which violates the derivation given above for a $4-\sigma$ sensitivity function. This may indicate a source of error which is outside the normal physical explanations. There may be "grey" areas in the data processing which can introduce substantial errors. For instance, unrecognized 3- $\sigma$ clouds, and possible continuum fitting errors may cause an overestimation of the continuum noise, and a correspondingly under-estimated significance level of a given cloud. There are four cases in which cloud EWs are significantly larger (in $m \AA$ ) than the flux-based sensitivity function at that location (3C 273, IZW1, Mrk 335, and Mrk 501) which may be explained by this logic. But there are a similar number in which their sensitivity functions are lower, and this cannot be explained in the same manner. The cause of this variation is uncertain. However, since the sensitivity functions based on the flux in the continuum is a simpler relation, the author judges that the flux-based sensitivity functions are more likely to reliably reflect the actual sensitivity. For this reason our standard processing of data uses flux-based sensitivity functions. The significance levels of the cloud are hence re-evaluated according to their EW relative to the sensitivity function. 


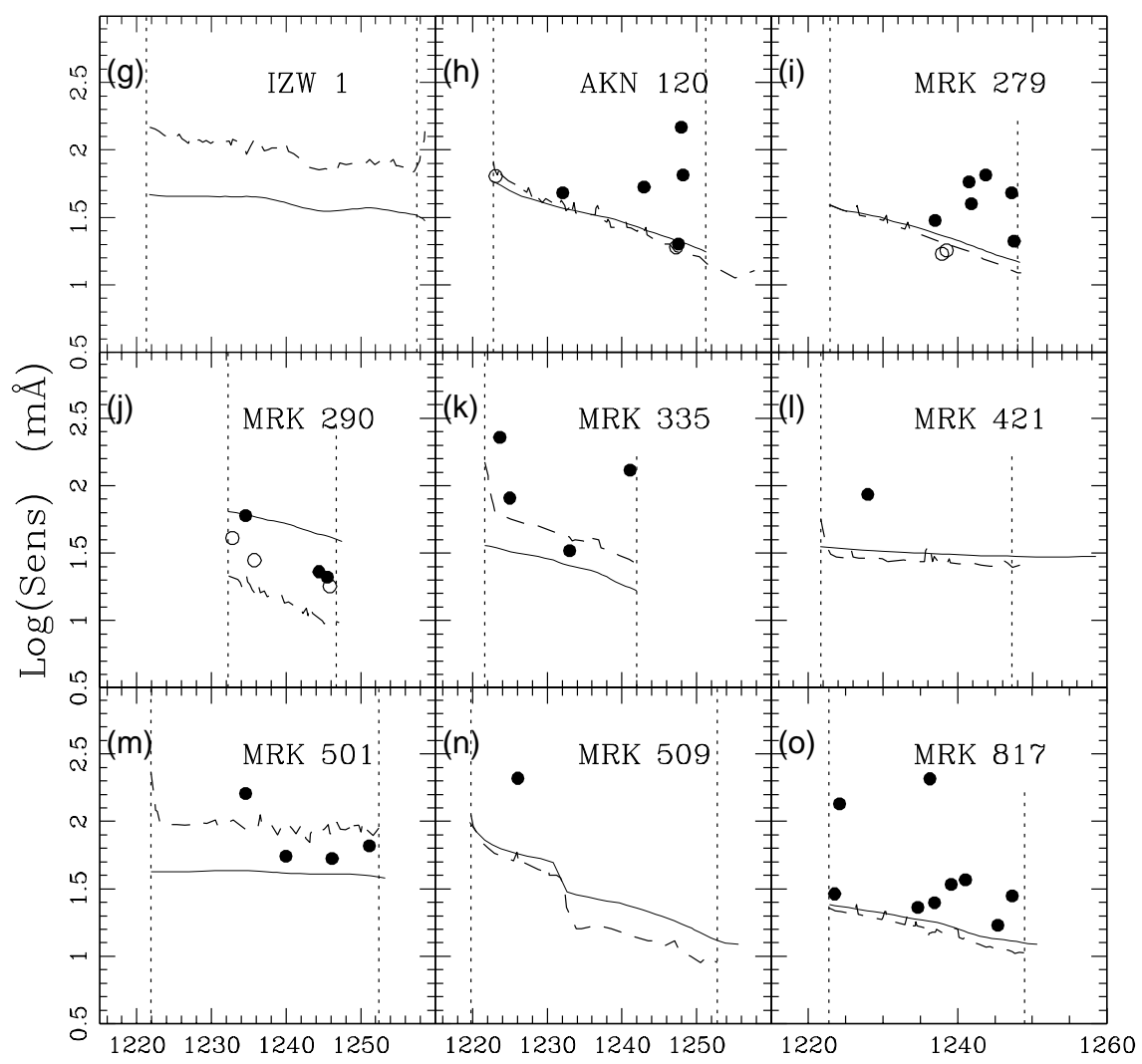

Fig. 2.- (Continued)

Wavelength $\lambda(\AA)$

\subsection{The Galaxy Catalogs}

A catalogue of galaxies is constructed along each LOS, and this is used this to calculate $\mathcal{T}$. All galaxy catalogs are taken from the CfA Redshift catalog, maintained by J. Huchra (Huchra et al. 1990, Huchra, Geller, \& Corwin 1995, Marzke, Huchra, \& Geller 1996, Grogin, Geller, \& Huchra 1998, Huchra, Vogeley, \& Geller 1999) ${ }^{3}$. The data which the catalog has that is of use to our enterprise is the position (B1950), velocity (with error), magnitude (with error), and the de Vaucouleurs T-Type. The T-Type is negative for early-type galaxies and quasars and positive for spirals and irregulars. This allows one to pick out those galaxies which are not well-described by the Tully-Fisher relation. The catalogs specific to each line of sight include all galaxies in the CfA catalog which are within a cylinder $7.5 \mathrm{~h}_{75}^{-1} \mathrm{Mpc}$ in radius, and within 15 degrees of the line of sight, and which have a redshift range extending $750 \mathrm{~km} \mathrm{~s}^{-1}$ beyond the spectral endpoints (see Table $1)$. We discuss the completeness levels of these catalogs in $\S 5.4$.

The radial positions of both galaxies and clouds relative to the observer are assumed to be that attributed on the basis of the redshift alone; clouds and galaxies are assumed to have a vanishing peculiar velocity (see $\S 5.5$ for further comment on this). Galaxy apparent magnitudes are assumed to be blue magnitudes, though the specific filter varies, and there is a low level of "contamination" with other filters when blue is not

\footnotetext{
${ }^{3}$ see http://cfa-www.harvard.edu/ huchra/
} 
available. The Tully-Fisher relation (Tully \& Fisher 1977, hearafter, TF) is used to determine the circular velocity range (see $\$ 5.1 .1$ ), and from this, the galaxy mass as a function of halo model. Though not all galaxies are spirals, we can adjust for this by noting that galaxies with negative de Vaucouleurs T-Types generally have mass-to-light ratios 2 to 4 times greater than spirals. We discuss this detail in $\S .6 .2$.

Much depends on the reliability of the apparent magnitudes in the assembled galaxy catalogs. For those interested in this level of detail, the Appendix offers a reconciliation of the CfA magnitudes with magnitudes extracted electronically from digitized Palomar Observatory Sky Survey plates, where we conclude that the CfA magnitudes are accurate to \pm 0.4 mag..

\section{The Galaxy Mass and Tidal Fields}

The next task is to calculate the tidal field produced by the galaxies in each catalog for each point along the lines of sight. We must first discuss the derivation of galaxy masses, then the tidal field. We also inspect the results of applying this methodology to the galaxy catalogs along the sightlines to AGN.

\subsection{Galaxy mass}

The basic procedure for deriving a galaxy's mass is as follows: apparent magnitude and redshift are used to derive an absolute magnitude, then an empirical relation must be exploited to estimate the galaxy mass. The overwhelming fraction of galaxies are of spiral type. For those, the TF relation is used to determine the circular velocity of the galaxy, and hence its mass as a function of the adopted halo model. The TF relation expresses the luminosity as a power of the circular velocity,

$$
\mathcal{L} \propto v_{c}^{\beta}
$$

where $v_{c}$ is the maximum circular velocity of the galaxy, and the slope $\beta$ is a function of spectral range. Tully \& Pierce (2000) show that the blue-band slope, relevant to the CfA catalogue, is $\beta_{B}=2.91$. The B-band galaxy luminosity is influenced by recent star formation, and so is more likely to experience larger excursions from the mean relation than, for instance, the K-band relation. For individual galaxies, there is a $0.55 \mathrm{mag}$ dispersion relative to the calibrated $B$-band relation (Tully \& Pierce 2000), but only 0.4 mag in $R$ and $K^{\prime}$. Given a DM halo model, one can extract a galaxy mass with error.

The derived mass is a function of the halo model. But having accurate relative masses of galaxies is more important at this stage than achieving accurate absolute masses. We consider two halo types. Our standard model is a truncated isothermal halo, and the NFW model, is treated as the first alternative. We treat the two halo models in order below.

\subsubsection{The mass of truncated isothermal spheres}

We first assume that the distribution of galaxy mass is well-modeled by a truncated isothermal sphere. In this case, the halo mass density can be approximated by,

$$
\rho(R)=\frac{\mathcal{K}}{4 \pi R^{2}}, \ldots \ldots \ldots \ldots \ldots . . .\left(R \leq R_{t}\right),
$$


where $\mathcal{K}$ is called the total mass distribution constant, and $R_{t}$ is the mass-truncation radius. Since the TF relation is based on circular velocities for dark matter plus baryons, $\mathcal{K}$ is taken to be the observed mass-distribution constant, where

$$
\mathcal{K}=\frac{v_{c}^{2}}{G}
$$

Assume that the galaxy masses are truncated at a radius such that the density at $R_{t}$ is the same for all galaxies. A glance at Eq. 27 will show that the truncation radius $R_{t} \propto \mathcal{K}^{1 / 2}$. With the above relations (Eqs 26,27 , and 28) we may construct the following scaling relations valid for isothermal halos:

$$
\frac{v_{c}}{v_{c}^{*}}=\left(\frac{\mathcal{L}}{\mathcal{L}^{*}}\right)^{1 / \beta_{B}}=\left(\frac{\mathcal{K}}{\mathcal{K}^{*}}\right)^{\frac{1}{2}}=\frac{R_{t}}{R_{t}^{*}}=\left(\frac{M\left(R_{t}\right)}{M^{*}\left(R_{t}\right)}\right)^{\frac{1}{3}},
$$

This halo model is standardized by requiring that an $\mathcal{L}^{*}$ galaxy has a truncation radius $R_{t}$ of $0.5 \mathrm{~h}_{75}^{-1}$ Mpc, consistent with the observations by Zaritsky \& White (1994) and Zaritsky et al. (1997) of the steadiness of velocity dispersions of satellite galaxies at large galactocentric radii around parent field spirals.

With the use of the cataloged redshift, the absolute magnitude of the galaxies is calculated. The TullyFisher relation is used to derive the circular velocity. The relevant error in absolute magnitude is the CfA magnitude error ( $\pm 0.4 \mathrm{mag}$, Appendix) combined in quadrature with the TF error per galaxy (0.55 mag in the $B$-band), which results in a total error of 0.68 mag. According to Tully \& Pierce (2000), the maximum circular velocity of a galaxy with absolute magnitude of $M_{B}$ is,

$$
v_{c}=\left[158 \exp \left(\frac{-\left(M_{B}+20.11-5 \log h_{75}\right)}{7.27}\right)\right]_{-0.19 v_{c}}^{+0.255 v_{c}} \mathrm{~km} \mathrm{~s}^{-1}
$$

where the 1- $\sigma$ errors in attributed total circular velocities are shown. The calibration of Tully (1988) indicates that $M_{B}^{*}=-20.18+5 \log \mathrm{h}_{75}$. The total mass $M^{*}$ of an $\mathcal{L}^{*}$ galaxy is then,

$$
M^{*}=\frac{\left(v_{c}^{*}\right)^{2} R_{t}}{G}
$$

where $v_{c}^{*}$ is the circular velocity of a $\mathcal{L}^{*}$ galaxy. With the scaling equations (Eq. 29) we see that $M \propto v_{c}^{3}$. The mass of a galaxy may be expressed as,

$$
M=\left[M^{*}\left(\frac{\mathcal{L}}{\mathcal{L}^{*}}\right)^{3 / \beta_{B}}\right]_{-0.35 M}^{+0.54 M},
$$

where $\mathcal{L} / \mathcal{L}^{*}=C \exp \left(-0.4 M_{B}\right)$, and $C=8.47 \times 10^{-9}$, and $\beta_{B}=2.91$. The $1-\sigma$ range in observed mass $M$ is then $0.65 M$ to $1.54 M$.

Note that if one sets $M_{B}=M_{B}^{*}=-20.18 \mathrm{mag}(h=0.75)$ (Tully 1988), one can solve for $v_{c}^{*}$ by substituting $M_{B}^{*}$ and using Eq. 30. In the B-band,

$$
v_{c}^{*}=161.5 \mathrm{~km} \mathrm{~s}^{-1} \text {. }
$$

What evidence, you might ask, is there to support this halo model (other than flat rotation curves)? Each halo at large galactocentric radius, and the gas associated with the halo, is assumed to scale as shown in Eq. 29, indicating that at a radius which scales $r \propto \mathcal{L}^{1 / \beta}$, the gas density of each system would be the same. 
We assume that the EW of HI gas found at distance $r_{p}$ from a galaxy should be proportional to the gas density there. A recent paper by Chen et al. (2001) has calculated the radius at which a characteristic absorption of $0.3 \AA$ occurs and found that in the K-band, $r \propto \mathcal{L}_{K}^{0.28 \pm 0.08}$ at a characteristic radius $r_{c h} \approx 180 \mathrm{~h}^{-1} \mathrm{kpc}$. In the B-band, $r \propto \mathcal{L}_{B}^{0.39 \pm 0.09}$. The isothermal halo model predicts that the exponent for both is the respective $\beta^{-1}$, which, given that $\beta_{K}=3.51$ (Cole et al. 2001), and $\beta_{B}=2.91$ yields $r \propto \mathcal{L}^{0.285}$, and $\propto \mathcal{L}^{0.344}$, both well within the errors quoted above. That is, the gaseous halos observed by Chen et al. (2001), and the scaling laws for isothermal halos, are consistent with each other.

On the other hand, the truncated isothermal halos proposed here are significantly more massive than those of the favored, NFW halo. While the mass within the distances probed in studies of the massluminosity ratio (e.g.. Bahcall, Lubin, \& Dorman 1995) is compatable with the NFW halo, so is the mass of an isothermal halo. It is in the realm beyond the virial radius, which has not been effectively probed, where this extra mass may be found.

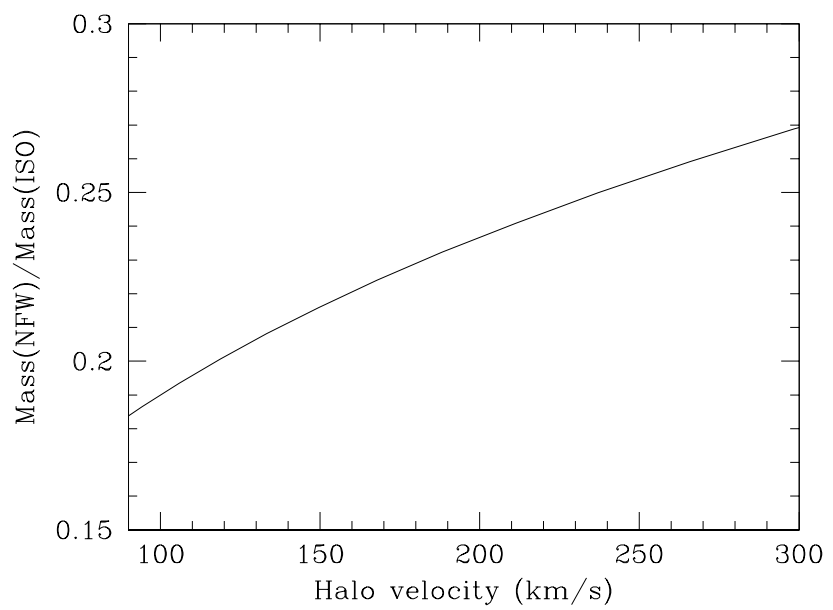

Fig. 3. - The ratio of the NFW virial mass to an isothermal halo truncated at a radius scaled to that of an $\mathcal{L}^{*}$ galaxy with $r_{t}=0.5 h_{75}^{-1} \mathrm{Mpc}$. The assumption of an isothermal halo will have the effect of attributing relatively more mass to small halos than to large. The skew from strict proportionality will be less than about $10 \%$ over the range of halo velocities of interest in this paper.

\subsubsection{The mass of a NFW halo}

If halos are described as NFW, then $\mathcal{K} \equiv M_{\max } / R_{\max }$, where $R_{\max }$ is the distance at which the circular velocity of the halo is a maximum. The density is,

$$
\rho(R)=\frac{\delta_{c} \rho_{\text {crit }}}{\left(R / R_{s}\right)\left(1+R / R_{s}\right)^{2}},
$$

(NFW, 1997), where $R_{s}$ is the scaling radius; $R_{\max } \simeq 2.16 R_{s}$. NFW halos are defined for the dark component only, and one has to adjust for the baryon fraction. This can be done by requiring that enough baryons are added within $r_{\max }$ to give a flat rotation curve inward of that point. As with the isothermal halo, the circular velocity is derived from the apparent magnitude and the redshift (refer to Eq. 30). The concentration parameter $c$ is defined as the ratio of the virial radius $R_{200}$ and the scaling radius $R_{s}$. 
By fitting the relation in Fig. 9 of NFW (1996) we find,

$$
\frac{v_{\max }}{v_{200}}=2.44 v_{\max }^{-0.110}
$$

Figure 10 in NFW (1996) gives,

$$
R_{\max }=1.5 \times 10^{-2} v_{\max }^{1.49} .
$$

From Eq. 3 of NFW (1997) we find a reasonable fit yielding,

$$
\frac{v_{\max }}{v_{200}}=\frac{c^{1 / 2}}{(2.16\{\ln (1+c)-c /(1+c)\})^{1 / 2}}=1.27 c^{0.28} .
$$

Equating Eqs. 39 and 41 yields,

$$
c=132.3 v_{\max }^{-0.393}
$$

Next we find,

$$
\frac{M_{200}}{M_{\max }}=\left(\frac{v_{200}}{v_{\max }}\right)^{2} \frac{R_{200}}{R_{\max }}=1.20 c^{0.44} .
$$

Using these equations we can construct scaling relations analogous to those of Eq. 29,

$$
\frac{v_{c}}{v_{c}^{*}}=\left(\frac{\mathcal{L}}{\mathcal{L}^{*}}\right)^{1 / \beta}=\left(\frac{R_{\max }}{R_{\max }^{*}}\right)^{0.67}=\left(\frac{R_{200}}{R_{200}^{*}}\right)^{0.76}=\left(\frac{M_{200}}{M_{200}^{*}}\right)^{0.30} .
$$

The errors propagate in the same way as with the isothermal halo though the virial mass is proportional to $v_{\text {max }}^{3.32}$, rather than $v_{\text {max }}^{3.0}$ for the isothermal halo. This accounts for the rising trend in the ratio of masses of NFW and isothermal halos of the same $v_{\max }$ (see Fig. 3).

How does this halo accord with the characteristic radius for absorption observed by Chen et al. (2001)? We would expect that it should scale with the virial radius. From the scaling relations above, $R_{200} \propto$ $\mathcal{L}^{1 /(0.76 \beta)}$, yielding $R_{200}(K) \propto \mathcal{L}_{K}^{0.375}$, and $R_{200}(B) \propto \mathcal{L}_{B}^{0.452}$, which are supposed to compare with exponents $0.28 \pm 0.08$, and $0.39 \pm 0.09$, respectively from Chen et al. (2001). The predicted $K$-band relation is outside the error bars, while the $B$-band relation is within the 1- $\sigma$ error bars, but near the edge. If instead one were to require the characteristic radius to scale with $\max _{\text {ax }}$, then the lack of concordance is exascerbated, giving exponents 0.43 and 0.52 for $K$ and $B$-bands, respectively, both well-outside $1-\sigma$ error bars of Chen et al. (2001).

The NFW halo, however, is defined by the dark component only. By adding baryons until a flat rotation curve is maintained out to $r_{\max }$, one finds that the circular velocity is increased by from $\sim 12 \%$, for small clouds, to $\sim 8 \%$ for large galaxies. The NFW halos are corrected for their baryonic part when comparing the masses of NFW and isothermal halos.

\section{2. $\quad$ Error in Attributed Tidal Fields}

We have seen in general terms how the tidal field is calculated along the LOS (§3.1). If we assign no error for the distance $R(i)$, the fractional error in individual tidal fields is the same as that in the attribution of mass to a galaxy on the basis of its redshift and apparent magnitude $m_{B}$ - that is, on order $50 \%$. The error in $R(i)$ will almost entirely result from positional errors along the line of sight (see §3.1). The fractional error in the attributed tide produced by a number of different galaxies will generally be significantly less 
than from a single galaxy because the individual errors are independent. However, since the masses and tides vary, there is no simple way to calculate the total error except by a specific convolving with redshift and mass errors of the effects of each galaxy. This level of sophistication is not pursued here, however, we will discuss the errors resulting from cloud peculiar velocities and redshift errors (§5.5).

Another source of error is the volume from which the galaxy catalogs are drawn. Let us consider the tidal field of an $\mathcal{L}^{*}$ galaxy at the edge of the $7.5 \mathrm{Mpc}$ cylinder at the same redshift of a cloud (a minimum maximum distance, so to speak). For an NFW halo the vector tide at $7.5 \mathrm{Mpc}$ is $\mathcal{T}_{R} \simeq 0.00195$, while the isothermal halo is $\sim 5$ times greater, $\mathcal{T}_{R} \simeq 0.0098$. This tide would produce a scalar tide of $\mathcal{T}=6.5 \times 10^{-4}$, and $3.3 \times 10^{-3}$ for the NFW and isothermal halos, respectively. Thus our estimated relative tidal fields may begin to produce serious errors at respective tides of this order. We shall see $(=$ S5.4, Fig. 5) that only a small fraction of galaxy mass is contained in galaxies brighter than $\mathcal{L}^{*}$, so the magnitude of the error is generally small.

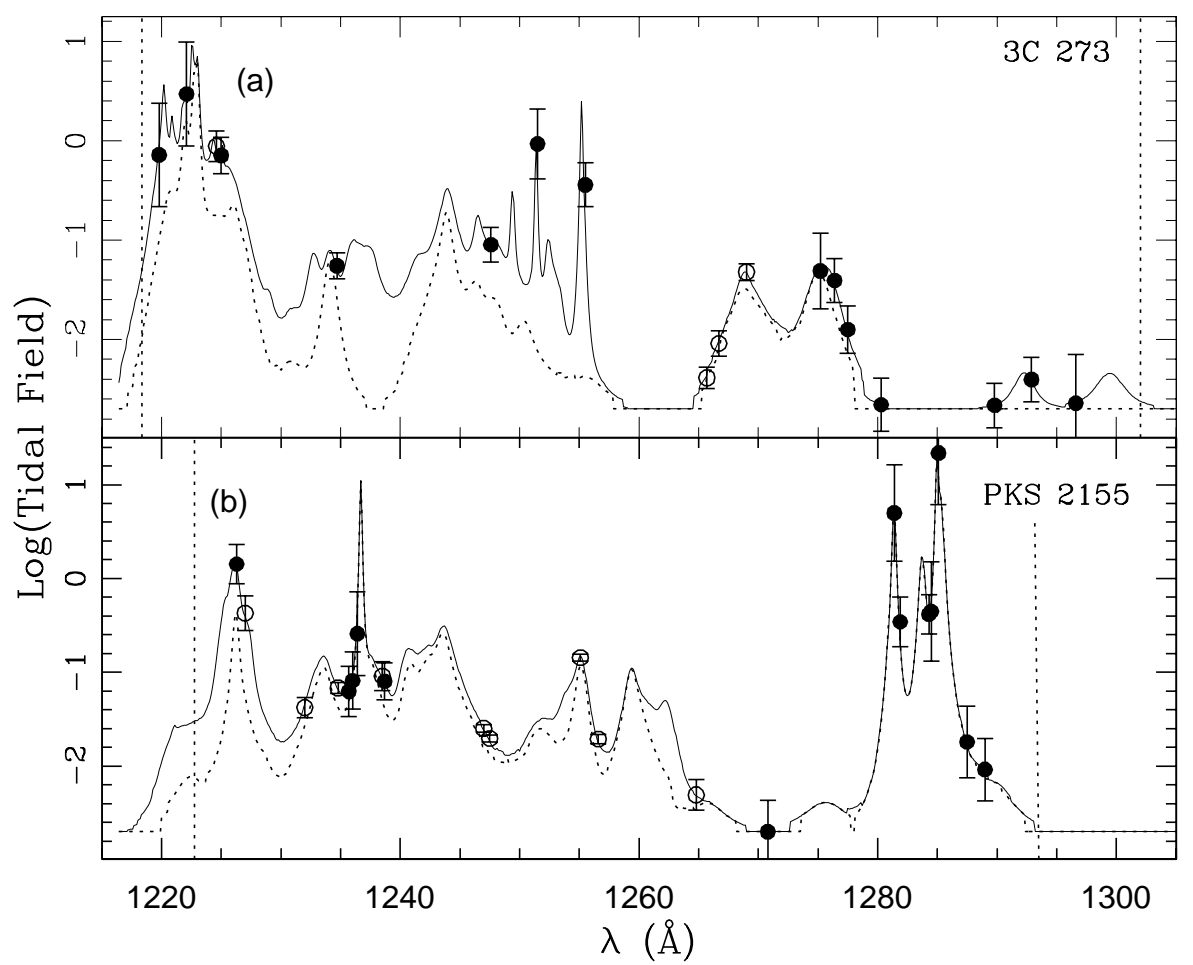

Fig. 4.- Tidal fields along the lines of sight to target AGNs. Panels $a$ and $b$ show 3c273 and PKS 2155-304. The tides are expressed in units of the tidal field parameter $\mathcal{T}$ (§3.1), which is convenient for constraining average cloud density for stability against disruption (see Eq. 19). The solid lines represent all galaxy data, while the dotted lines represent the effects of only those galaxies brighter than $M_{B}=-19.8 \mathrm{mag}$, the absolute magnitude of a $m_{B}=16$ galaxy at $z=0.036$ (see $\S 5.2$ ). The highly significant (4- $\sigma$ ) absorption systems, in terms of the author's sensitivity functions (solid lines, Fig. $2 a-o$, are denoted by the solid circle), while the 3- $\sigma$ clouds are represented by open symbols. The cloud EWs are denoted by the error bars, with length proportional to $\log \mathcal{W}$. The vertical dotted lines show the limits of the useful portion of the absorption system surveys (see $\S 3.1$ and Table 1 ). 


\subsection{Sightline Characteristics}

As mentioned above ( $\$ 5.2)$, the LOS component of the tidal field is calculated assuming a pure Hubble flow of galaxies, while those normal to the LOS are calculated using the appropriate angular diameter distance relation. Figure 4 shows the calculated tidal fields (solid line) as a function of position along the 15 LOS. Cloud positions are noted by the circles (filled, $S L \geq 4-\sigma$, open, $S L<4-\sigma$ ) with vertical bars, whose lengths are proportional to $\log \mathcal{W}$. Note the similarity of the tidal fields in the sightlines to $3 \mathrm{C} 273$ and Q1230+0155, which both pass through the Virgo cluster. Some stretches of space in the various lines of sight have no cataloged galaxies within the search criteria, and so have a formally zero tide. A value of 0.002 is added to all tides so that the line would appear on the plots. The significance of the dotted lines which "shadow" the tidal field traces is discussed in the next subsection.

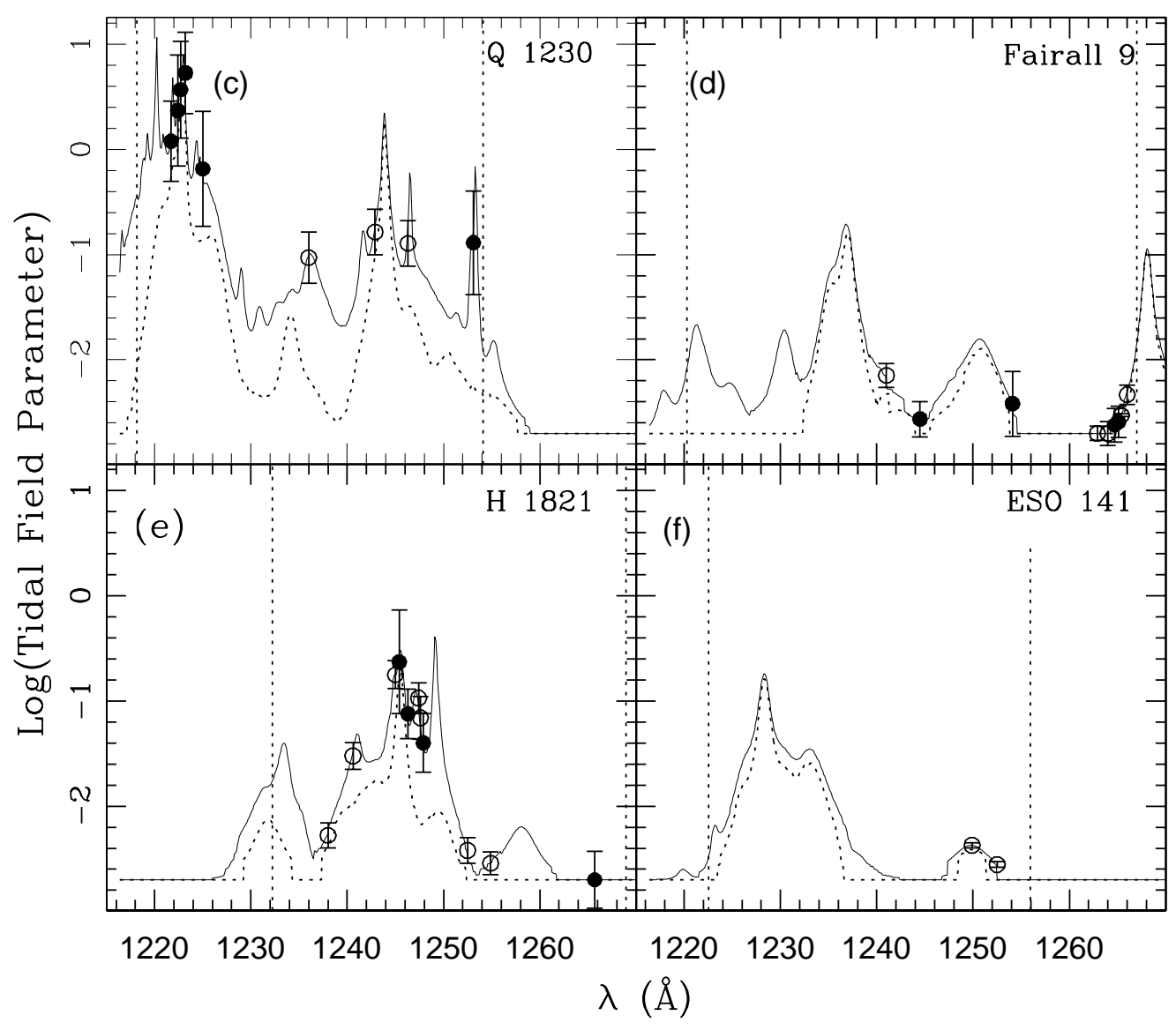

Fig. 4.- (Continued)

\section{4. $\quad$ Effect of Lack of Completeness}

Though galaxy detections are surface brightness selected, the decision to extract a redshift and place an object in a galaxy catalog are often determined by the total apparent magnitude. The CfA catalog is 


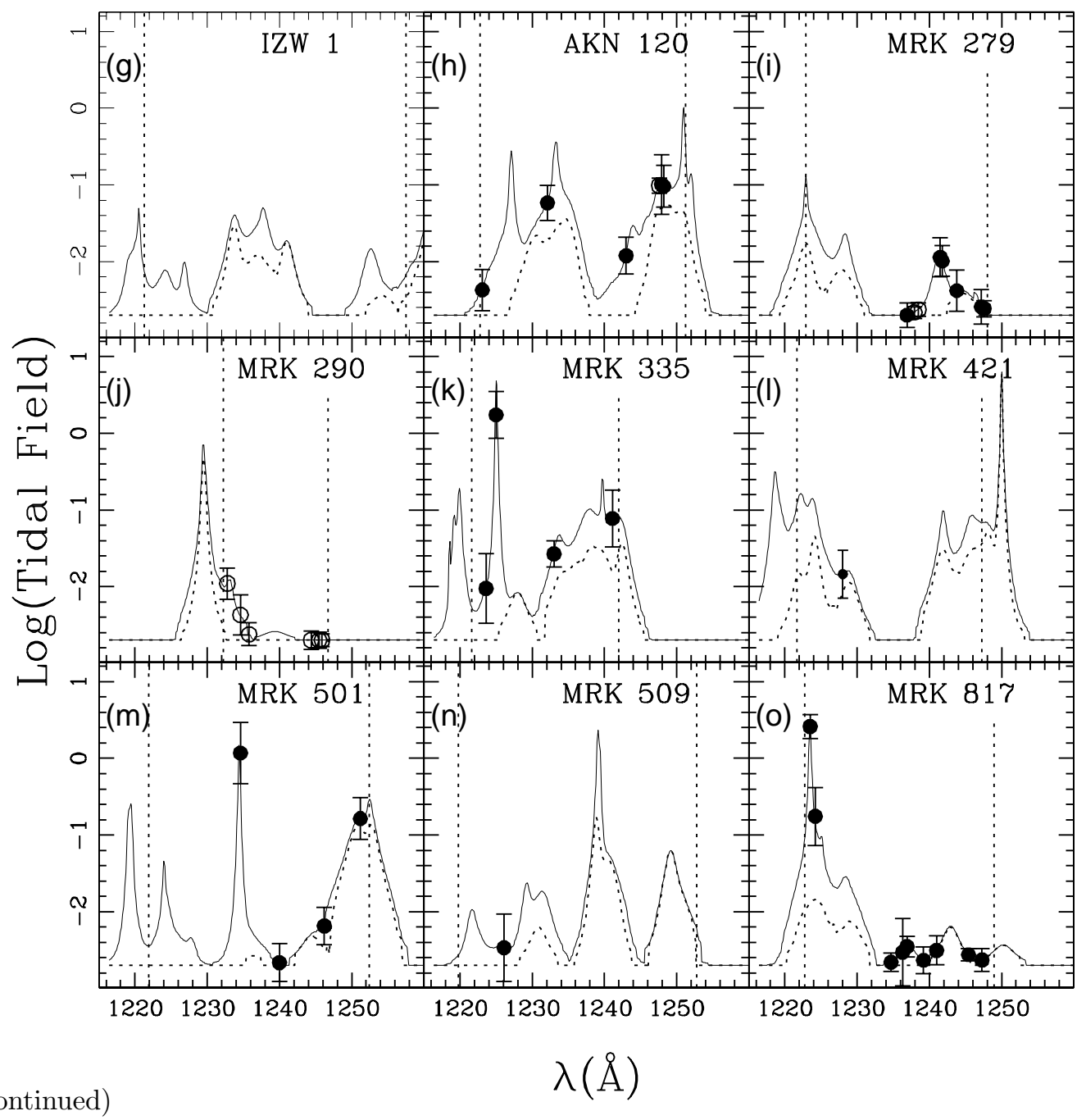

Fig. 4.- Continued)

generally based on Zwicky magnitudes and have a completeness limit of order $15.5 \leq m \leq 16.0 \mathrm{mag}$, but the catalog accepts galaxy data from a wide range of sources. To get an idea of how much mass is unrepresented in the catalogs, a fractional cumulative mass function is calculated. Figure 5 shows the cumulative fractional mass function for the truncated isothermal halo in terms of the absolute magnitude of the galaxy with a faint-end slope $\alpha=-1.2$ (solid line). It is produced by the following integral,

$$
f_{M}\left(<v_{c}\right)=\frac{\int_{v_{i}}^{v_{c}} M\left(v_{c}\right) \phi\left(v_{c}\right) d v_{c}}{\int_{v_{i}}^{v_{f}} M\left(v_{c}\right) \phi\left(v_{c}\right) d v_{c}},
$$

where $v_{i}=37 \mathrm{~km} \mathrm{~s}^{-1}$ and $v_{f}=375 \mathrm{~km} \mathrm{~s}^{-1}$, which encompasses the overwhelming majority of galaxian mass, and $\phi(v)$ is the B-band luminosity function transformed to a velocity function by use of the TF relation. With the use of Eq. 30 we express halo velocity in terms of absolute magnitude. Note that $\sim 35 \%$ of the galaxy mass is in the range $M_{B} \lesssim-19.8$ mag. A galaxy with $M_{B}=-19.8$ is $0.74 \mathcal{L}^{*}\left(v_{c} \sim 146 \mathrm{~km} \mathrm{~s}^{-1}\right)$, and has an apparent magnitude of 16 mag at $z \simeq 0.036$. 
However, it may be reasonable to suggest that the sub-halos within a given halo are characteristic of the system, and not an excess over the halo attributed to the luminosity of the central galaxy. The NFW halo is the profile for an individual halo. Studies of "secondary" infall (Zaroubi \& Hoffman 1993; AvilaReese et al. 1999; Klypin et al. 1999) suggest that sub-halos are not included in the mass distribution of the standard NFW halo. The truncated isothermal halo, however, describes a system extending far beyond the galaxy which has formed at its center. In this case, the sub-halos are interpreted as an aspect of the system referred to by the mass distribution constant $\mathcal{K}$ (see Eq. 28). So in the case of the isothermal halo, we count sub-halos twice unless a factor representing the fraction of halos with a given halo velocity which are not sub-halos is inserted into both upper and lower integrals of Eq. 41. The fraction of halos of velocity $v_{c}$ which are isolated has been estimated by Avila-Reese et al. (1999), and at low $v_{c}$ it appears to follow the relation $f_{\text {iso }}=1.36\left(v_{c} / 225\right)-0.23$ for halos smaller than $158 \mathrm{~km} \mathrm{~s}^{-1}(0.7 \times 225)$. The results of applying $f_{i s o}$ to the the numerator and denominator of Eq. 41 is the dotted line in Fig. 5. While with the former $M_{B}=-19.8 \mathrm{mag}$ occurs at $f_{M} \simeq 0.65$, with the latter it occurs at $f_{M}=0.44$. The implication of this is that the sub-halos of the brighter galaxies are counted already, so less is missed. On the other hand, it now appears possible that we may have over-estimated the tidal field at very low-redshift by observing their sub-halos, and thereby effectively counting them twice. There is thus an inevitable tendency to find more mass at low redshift. Above $z \sim 0.036$, about half of the mass in galaxies is missed for catalogs with a uniform magnitude limit $m_{B}=16$.

Four spectra extend above $z=0.036$, 3C 273, PKS 2155-304, Fairall 9, and H1821+643 with a total of 21 clouds. To test for the effects of incompleteness one could calculate the equivalent width distribution with, and without, the data from $z \geq 0.036$. If there is no significant difference, then one might say that completeness issues are not debilitory to the conclusions of the paper, for if no completeness effects found by the removal of data with $z \geq 0.036$ then it is unlikely to do so at lower redshifts. However, we should expect that clouds at higher redshift may have calculated ambient tidal fields a factor of $\simeq 2$ low, hence one runs the risk of placing what may be GDS clouds in void catalogs.

A sense of the potential effects of incompleteness can be seen by placing a uniform limit on the luminosity of galaxies used to calculate tides. In Fig. 4 (panels a-o) the dotted line represents the tidal field when galaxies with $M_{B}$ fainter than $-19.8 \mathrm{mag}$ are removed from consideration. Generally the discrepancy between the total and the contribution from only the brighter galaxies declines with increased redshift as fewer and fewer low luminosity galaxies make the catalog (a good example of this is panel $b$ ). This is characteristic of fields of roughly uniform apparent magnitude limits. On the other hand, the sightline to 3C 273 (panel $a$ ) has regions at high-redshift where removing galaxies less luminous than $M_{B}=-19.8$ has a strong effect on the calculated tidal field. This is because some effort has been expended to get the redshifts and magnitudes of galaxies along the line of sight (Morris et al. 1993), with a claimed completeness to $m_{B}=19.0$, so that one ought to be able to detect a galaxy of $M_{B}=-17.0$ at $z=0.065$.

\subsection{Attributed Positional Errors}

For clouds associated with galaxies, there are two major sources of error in the assignment of a value for $\mathcal{T}$ at the cloud's location, galaxy redshift errors, and peculiar velocities, particularly of the cloud. We discuss these in order below.

In this paper the main concern is with void clouds, which are only slightly effected by galaxy redshift errors since typical redshift errors, on order $20-30 \mathrm{~km} \mathrm{~s}^{-1}$, imply modest positional errors relative to void 


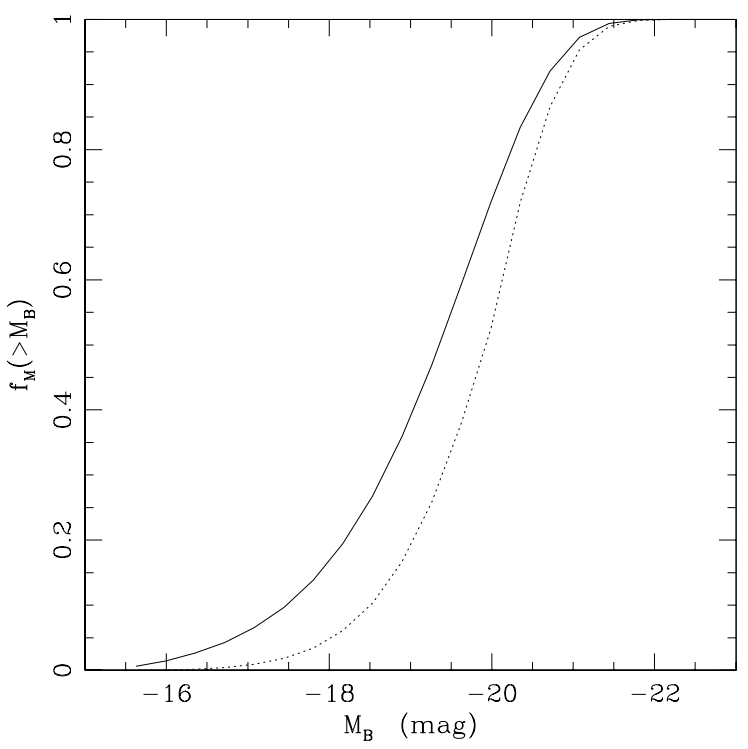

Fig. 5.- The normalized mass function as extracted from the Schechter Luminosity function (using $\alpha=$ -1.2 ) and the TF relation (solid line), both in the B-band. The dotted line is the mass function corrected for sub-halos (see $\S 5.4$ ).

clouds ( 260 to $400 \mathrm{kpc})$.

Cloud peculiar velocity errors stem from the "triple-value ambiguity" noted by Tully \& Shaya (1984) in which a given LOS velocity may occur at three different distances when the object is infalling toward a larger mass. If clouds are responding to the peculiar gravity of a galaxy, they may acquire maximum peculiar velocities not much greater than $v_{p} \approx v_{c}=\sqrt{G \mathcal{K}}$ (see Eq. 28) when the clouds are close to the galaxy ${ }^{4}$. A peculiar velocity of this order, under a strict Hubble flow, results in an attributed distance, $R_{\sigma}=\sqrt{G \mathcal{K}} / H_{0}$. The average over-density within this distance is,

$$
\bar{\rho}\left(R_{\sigma}\right)=2 \rho_{\text {crit }} \frac{G \mathcal{K} R_{t}}{H_{0}^{2} R_{\sigma}^{3}}=2 \rho_{\text {crit }}\left(\frac{R_{t}}{R_{\sigma}}\right) .
$$

For the Galaxy (assume $v_{c}=220 \mathrm{~km} \mathrm{~s}^{-1}$ ), the truncation radius $R_{t}$ is scaled up from $R_{t}^{*}=500 \mathrm{~h}_{75}^{-1} \mathrm{kpc}$ to $684 \mathrm{kpc}$. The over-density within radius $R_{\sigma}$ is $\bar{\rho} \simeq 0.46 \rho_{\text {crit }}$, where in this case, $R_{\sigma} \simeq 3.0 \mathrm{~h}_{75}^{-1} \mathrm{Mpc}$. The median position angle (the angle between the LOS and the vector from cloud to galaxy) for clouds distributed isotropically about a galaxy is $60^{\circ}$, leading to the conclusion that the average LOS velocity will be $\sim 1 / 2$ of $v_{p}$. Therefore, low cloud velocities relative to a galaxy suggest either that the cloud is distant, or that its velocity has dissipated against the gaseous halo surrounding the galaxy.

It is also possible that clouds which are actually distant (say, 2 to $3 \mathrm{Mpc}$ from a large galaxy), but have a small impact parameter, may appear at very nearly the same velocity as the galaxy; a slight peculiar velocity may balance the Hubble flow if the cloud is near the turnaround radius. Thus, clouds which are within $R_{\sigma} \simeq \sqrt{G \mathcal{K}} / H_{0}$ of a galaxy may not have their positions accurately predicted by the redshift due to the peculiar velocity. Such a huge positional error is not likely for the average cloud in GDS since it would

\footnotetext{
${ }^{4}$ If peculiar velocities are a measure of the galaxy potential, then with the truncated isothermal halo, the peculiar velocity will be of order $v_{p} \simeq 2^{1 / 2} v_{c} \sqrt{R_{t} / R}$.
} 
require a low-probability projection of the cloud at large radius $R \approx 3 \mathrm{Mpc}$ (where the cloud density is low) to a small projected radius $R_{p}$. However, again, this kind of error cannot substantially effect the position of a typical void cloud, nor its attributed $\mathcal{T}$.

Small groups of galaxies may, by their virial velocities, introduce error into their attributed positions. We have seen that voids may be considered to begin at distances of $\approx 2 \mathrm{Mpc}$ from a single $\sim \mathcal{L}^{*}$ galaxy $(\S 2.1)$, but that the distance at which a cloud is at the same degree of isolation from a group will be larger. Bahcall \& Cen (1993) show that the one-dimensional velocity dispersion in groups and clusters can be approximated by

$$
\sigma_{L O S} \approx(90 \pm 20) N_{g}^{1 / 2} \mathrm{~km} \mathrm{~s}^{-1},
$$

where $N_{g}$ is the number of galaxies in the group. For $N_{g}=10$, we have $\sigma_{L O S}=284 \mathrm{~km} \mathrm{~s}^{-1}$, or a spread of $\delta r \sim 1.9 h_{75}^{-1} \mathrm{Mpc}$ on either side of the center. But the scaling relations (Eq. 29) show that the radius at which a given $\mathcal{T}$ would occur is $\sim 10^{1 / 3}$ times farther than for a single galaxy. If we consider $2 \mathrm{Mpc}$ as a plausible minimum distance from a major galaxy for a void to begin (at $2 \mathrm{Mpc}$ from an $\mathcal{L}^{*}$ galaxy the tide $\left.\mathcal{T}_{R}=0.24\right)$, then this translates to a distance $\sim 4.3 \mathrm{Mpc}$ for the group. If the group is at the same redshift as the cloud, most of the group galaxies would have attributed radial positions within an angle of $\lesssim 24^{\circ}$ of the center of mass, as seen from the cloud's position, producing a distance variation of less than $\sim 9 \%$. The tidal field at a distance of $4.3 h_{75}^{-1} \mathrm{Mpc}$ from this group would be about $86 \%$ of that calculated assuming its galaxies were located at the group center. This error is significantly less than the error in an individual galaxy. Further, since the real apparent magnitudes of the group should differ randomly, the error in the tidal field at the cloud should have a lower value from apparent magnitude error than a cloud subject to the influences of just a few. An error of this magnitude is insignificant in the present analysis.

\subsection{Tides and Cloud Statistics}

Sightline analysis results in a value for tidal field $\mathcal{T}(\lambda)$ along each LOS (Fig. 4). The value of the specified tidal field limit separates the cloud population into two catalogs - those defined by $\mathcal{T}$ as an upper limit $\mathcal{T}^{U}$, and those with $\mathcal{T}$ as a lower limit $\mathcal{T}^{L}$. Histograms of clouds for various $\mathcal{T}$ are presented in Fig. 6, showing the former, $\mathcal{C}\left(\mathcal{T}^{U}\right)$, as dotted lines, and the latter, $\mathcal{C}\left(\mathcal{T}^{L}\right)$, as solid lines. In these plots, what is referred to as "void" is relative to the current choice of $\mathcal{T}^{U}$. Thus, "void" is relative to the stated tidal field limits. If a corresponding physical distinction between void and GDS clouds can be found, then "void" may take on a more absolute meaning. Note that the void histograms have a most probable Doppler parameter of $b=30$ to $35 \mathrm{~km} \mathrm{~s}^{-1}$ over the whole range of $\mathcal{T}^{U}$. The high-velocity wing of the distribution suggests the presence in voids of more massive clouds, while the cutoff at low $b$ gives the distribution a large skewness, with values ranging from 0.87 to 1.06 in voids $0.01 \lesssim \mathcal{T}_{\text {lim }} \lesssim 0.1$. For clouds defined by a lower bound $\mathcal{T}^{L}$, as one increases $\mathcal{T}^{L}$, the flat distribution apparent for lower values of $\mathcal{T}^{L}$ gives way to a peaked histogram and most probable Doppler parameter of $b \simeq 60 \mathrm{~km} \mathrm{~s}^{-1}$ for $\mathcal{T} \geq 0.3$. The skewness at $\mathcal{T}^{L}=0.3$ is -0.46 .

In Fig. $7 a$, the fractional redshift coverage as a function of tidal field upper limit has been equated to a fractional volume which has $\mathcal{T}<\mathcal{T}^{U}$. Notice that $90 \%$ of the universe has $\mathcal{T} \lesssim 0.16(\log \mathcal{T} \lesssim 0.8)$. Fig. $7 b$ shows the fractional redshift coverage as a function of $\mathrm{EW}$

It is of interest to attempt to determine the point $\mathcal{T}^{V}$ which actually separates void from GDS space. Figure 8 shows a differential histogram extracted from Figs. 6, which shows the number and b-value of clouds added to the void population in the stated interval. Panel $8 a$ shows a distribution quite similar to the overall void population; fig. $8 b$ shows a relatively flat distribution; Fig. $8 c$ shows a distribution with 


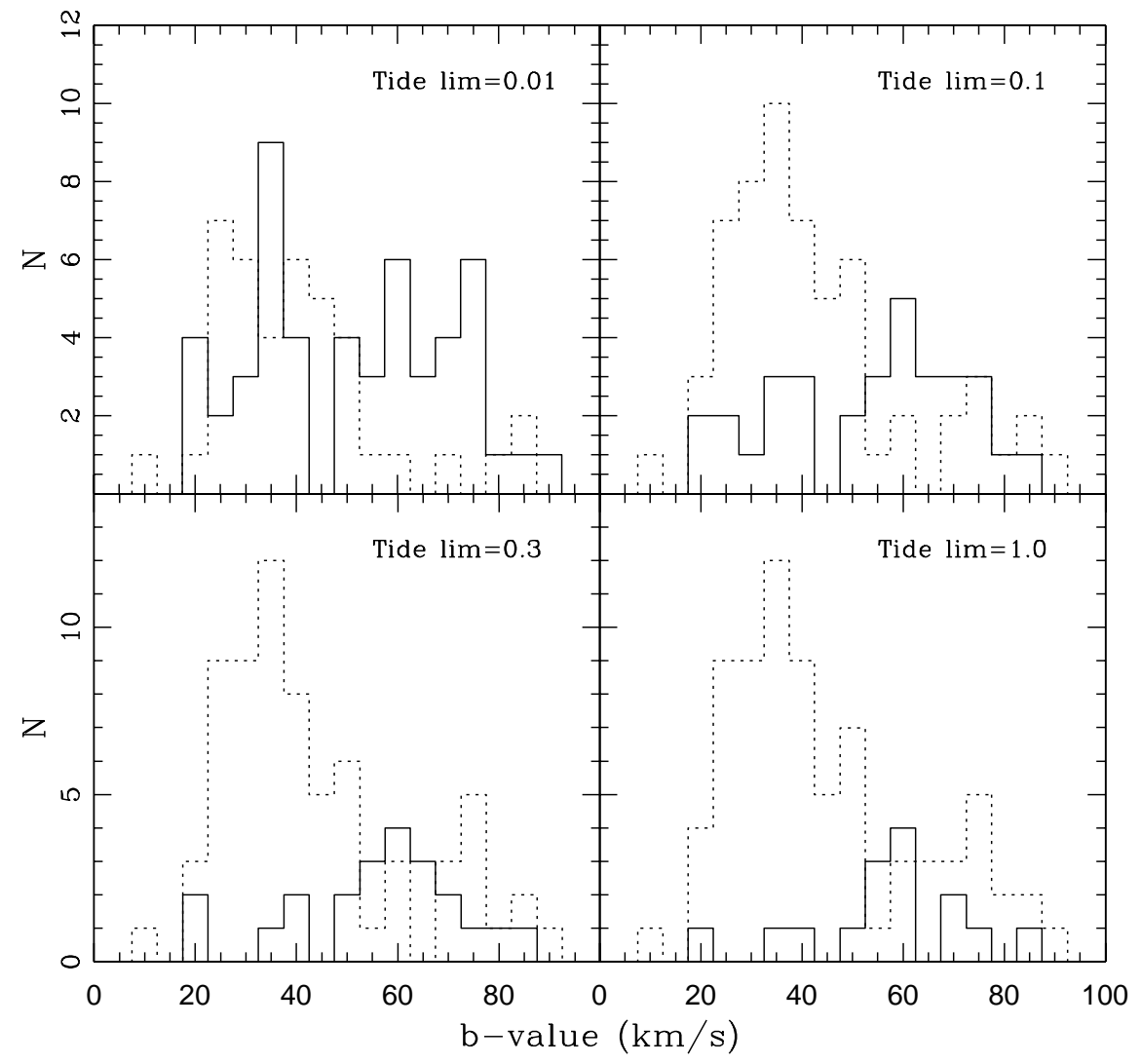

Fig. 6.- Histograms of void (dotted) versus non-void (solid) clouds as a function of cloud Doppler parameter for various $\mathcal{T}_{\text {lim }}$. "Void" is defined by the value of the current tidal field upper limit $\mathcal{T}^{U}$. Notice that the shape of the void cloud distribution remains much the same (though its size necessarily gets larger) as $\mathcal{T}^{U}$ increases until $\mathcal{T}_{\text {lim }} \simeq 0.3$.

characteristics of the GDS population. This implies that the break between void and GDS environments is at $0.1 \leq \mathcal{T} \leq 0.3$, which brackets $\mathcal{T}=0.16$, the tide at which $90 \%$ of the universe has a lower tide. Recall that 0.9 is the filling factor for the unshocked population ( $(1)$. Thus we provisionally assign $\log \mathcal{T}^{V}=-0.8 \pm 0.2$ as the contour dividing void and GDS space for ISOT halos, bracketing a void filling factor $0.87 \leq f_{v} \leq 0.84$.

\section{The Equivalent Width Distribution Functions}

In $\S 3$ it was shown that the likely effect of tides on a population of uniform clouds is to disrupt those with density $\rho \lesssim 2 \mathcal{T} \rho_{\text {crit }}$ (Eq. 19). However, clouds bound by dark matter are not expected to be of uniform density (see $\S 2$ ). Because of this, it is reasonable to suppose that the effect of tides on the distribution of absorber EWs may have its greatest observable effect on the slopes of the EWDF.

We have seen that the redshift coverage at low sensitivity is restricted. The EWDF removes this observational bias. The EWDF of clouds of a catalog $\mathcal{C}$ is calculated as the redshift frequency of clouds with rest-equivalent width greater than $\mathcal{W}$, directly for each equivalent width limit $\mathcal{W}$ by noting the number of clouds of any equivalent width greater than $\mathcal{W}$ within the ranges of wavelengths for which the sensitivity 


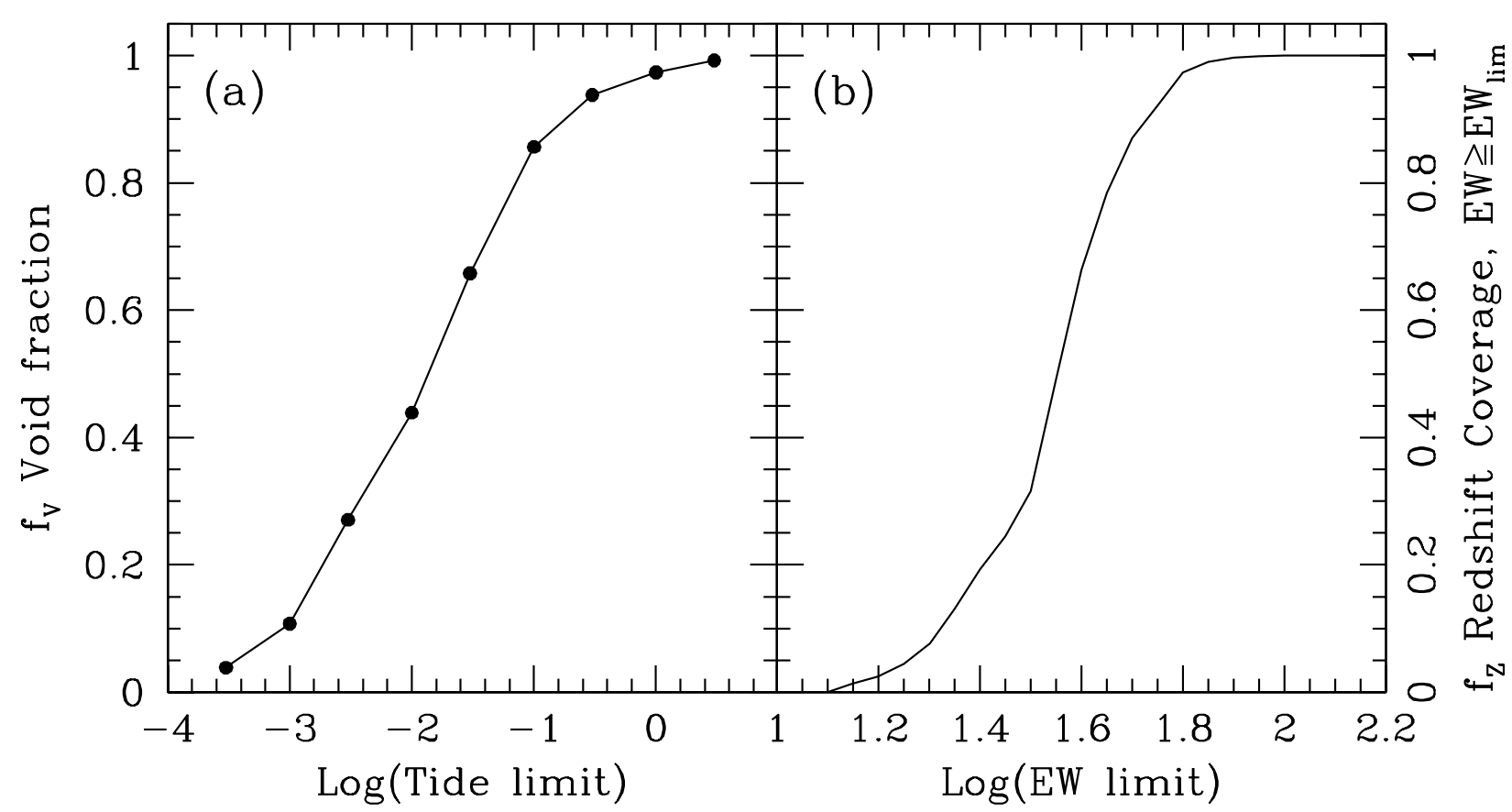

Fig. 7.- Panel (a): The fraction of redshift space with $\mathcal{T} \leq \mathcal{T}_{\text {lim }}$ plotted against $\log \mathcal{T}_{\text {lim }}$. This fraction can be considered to be a fair approximation of the volume filling factor for voids defined by $\mathcal{T} \leq \mathcal{T}_{\text {lim }}$. Note that tides $\log \mathcal{T} \lesssim-2.5$ may seriously underestimate the relative tidal field, as $\mathcal{L}^{*}$ galaxies farther than 7.5 Mpc may produce scalar tides of this order (see $\$ 5.2$ ). Panel (b): The fraction of redshift space with the sensitivity function (in $\mathrm{m} \AA$ ) less than $\mathcal{W}_{\text {lim }}$ as a function of $\log \mathcal{W}$ (in $\mathrm{m} \AA$ ).

functions have values less than $\mathcal{W}$. This is then divided by the summed redshift range for which the sensitivity $\mathcal{S}$ (in $\mathrm{m} \AA$ ) is smaller than $\mathcal{W}$ (Fig. 7b). Thus, the cumulative EWDF is,

$$
f(\mathcal{W})=\frac{d \mathcal{N}(\geq \mathcal{W})}{d z}=\frac{\sum_{\mathcal{W}}^{\infty} n_{c l}\left(\mathcal{W}_{c l} \geq \mathcal{W} \geq \mathcal{S}\right)}{\sum_{\mathcal{W}}^{\infty} \Delta z(\mathcal{W} \geq \mathcal{S})}
$$

where it is read, " $n_{c l}$ such that $\mathcal{W}_{c l} \geq \mathcal{W} \geq \mathcal{S}$ ", and " $\Delta z$ such that $\mathcal{W} \geq \mathcal{S}$ ". Note that while this distribution is ostensively cumulative, it is not impossible for the distribution to decline at low EW since there may be conditions in which the number of clouds in the redshift interval with very low sensitivity $\mathcal{S}$ is inordinately small. So while this distribution is cumulative, its calculation at each $\lambda$ is to some extent independent of its neighboring bins.

Results are presented below.

\subsection{Standard Processing}

Standard processing uses the ISOT model, accepts all 4- $\sigma$ clouds, and attributes galaxy mass based on apparent magnitude $m_{B}$, a flat- $\Lambda$ model, and the TF relation pertinent to the $B$-band. Fig. $9 a$ shows standard processing for all data irrespective of tidal field (which we hereafter refer to as "mean"), shown as the heavy solid line. Also shown, closely associated, is the mean EWDF taken from PSSI (dashed line). The 


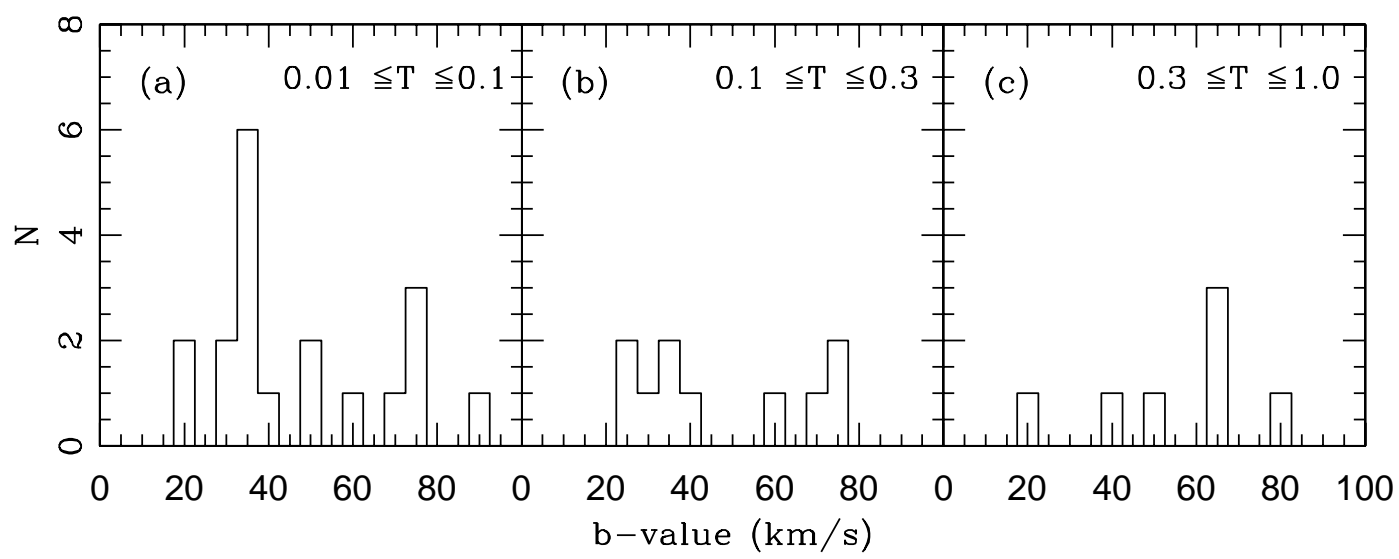

Fig. 8. - The differential histograms from Fig. 8; histograms of clouds added to $\mathcal{C}\left(\mathcal{T}^{U}\right)$ for $a \mathcal{T}^{U}=0.01$ to $0.1, b \mathcal{T}^{U}=0.1$ to 0.3 , and $c \mathcal{T}^{U}=0.3$ to 1.0 . The clouds added in panel (a) appear to have roughly the same distribution as is found for $\mathcal{T}^{U}=0.01$ in panel (a) of Fig. 8. Panel $b$ is distinctly flatter indicating that clouds which are non-void may be beginning to enter the population. Panel $c$ begins to show the distribution characteristic of non-void clouds, with a most likely Doppler parameter near $60 \mathrm{~km} \mathrm{~s}^{-1}$. This trend shows that the tidal field level at which the character of added void clouds experiences rapid change is at $0.1 \lesssim \mathcal{T} \lesssim 0.3$.

two are very close except at $\mathcal{W} \lesssim 80 \mathrm{~m} \AA$; where the PSSI shows a broad bump, the present EWDF shows an inflection to a higher slope. This difference is due to the different sensitivity functions employed in the present analysis. Also shown (the dotted line) is the EWDF for $\mathcal{T} \leq 0.1$, where the data used excluded the range $z>0.036$. Notice that its slope appears to be continuous with that of the inflected part of the mean EWDF (solid line). This is what one would expect if the low-EW part of the mean EWDF is dominated by clouds in voids. Also shown (thin solid lines) are the number-weighted least squares linear fits to the EWDFs.

In Fig. $9 b$ we show the Poisson errors associated with points for the mean EWDF (heavy solid) and the EWDFs formed by the complementary catalogs defined by $\mathcal{T}_{\text {lim }}=0.01$; GDS is above, void is below. The error bars are not independent, but simply reflect the number of clouds at that EW which are contributing to the EWDFs. The cutoffs evident in the EWDFs at high EW are probably due to the tendency of large EW clouds to have associated metal-line absorption, and so be rejected from the line lists. The cutoff in the $\mathcal{C}\left(\mathcal{T}^{L}\right)$ EWDF (the upper line) at low EW is the result of the paucity of low EW clouds in higher tidal field environments. The error bars show that the two complementary populations are significantly distinguished.

The processing of the EWDFs was done for a series of $\mathcal{T}_{\text {lim }}$ equally spaced in $\log \mathcal{T}$. Number-weighted linear fits to the equation,

$$
\log (d \mathcal{N} / d z)=\log f(\mathcal{W})=C+S \log (\mathcal{W} / 63),
$$

are calculated, where $C$ is the intercept $\log (d \mathcal{N} / d z)$ at $\mathcal{W}=10^{1.8} \simeq 63 \mathrm{~m} \AA$ (the approximate median $\log \mathcal{W}$ ) in the total cloud catalog, and $S$ is the slope. These results are presented in Table 2. For comparison, the fits for the mean EWDF is also presented. Because of the break in the distribution apparent at $\mathcal{W} \simeq 32 \mathrm{~m} \AA$, the fit is calculated including, and excluding the region $12 \leq \mathcal{W} \leq 32 \mathrm{~m} \AA$, respectively

The data from the lower two subdivisions of Table 2 are plotted in Fig. 10, where panel $a$ shows the trend of slope $S$ with $\log \mathcal{T}$, and panel $b$ shows the intercepts $C$. Slope fitting errors are large compared to 


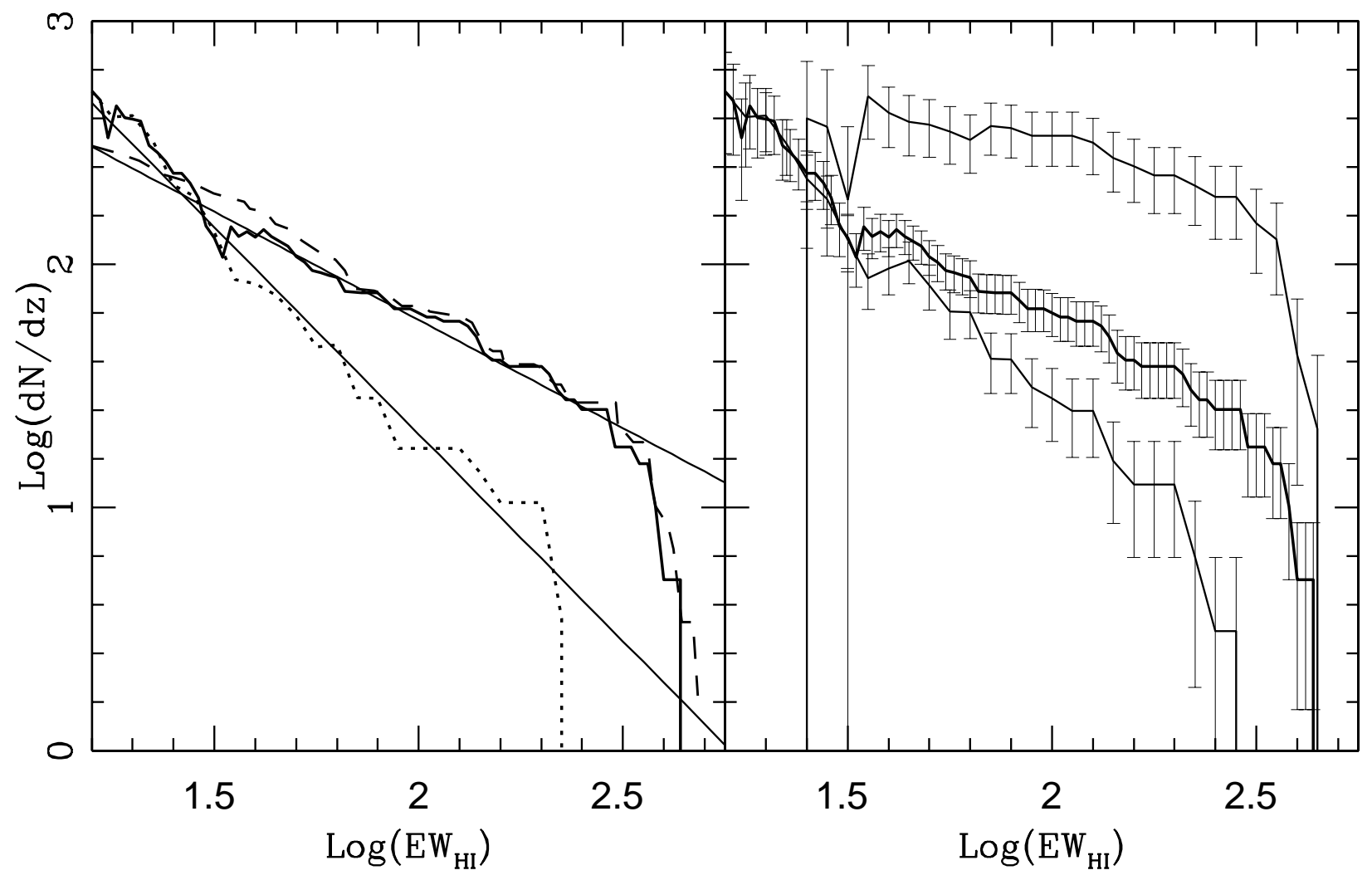

Fig. 9.- Equivalent width distribution functions, expressed as the logarithm of the cumulative line density as a function of the $\log$ of the $\mathrm{EW}$ (in $\mathrm{m} \AA$ ). Panel $a$, an analysis of the derived mean EWDF (heavy solid line), and that derived by PSSII (heavy dashed line), and the EWDF for $\mathcal{C}\left(\mathcal{T}^{U}\right)=0.01$ for $z<0.036$ (dotted). Thin lines are number-weighted linear fits to current data. Note the inflection in our mean EWDF (heavy line) at $\log (E W)=1.5$, which is not seen in Penton et al. analysis, is continuous with the void EWDF. Panel $b$, EWDFs with Poisson errors. The mean distribution function (heavy solid line), and the lighter lines are the EWDFs for catalogs formed using $\mathcal{T}_{\text {lim }}=0.1$ as lower-bound (upper line) and upper-bound (lower line).

intercept errors. The trends in Fig. 10 indicate that in higher tidal field environments the redshift frequency of absorption systems is larger, and the population more uniform in their EWs, while for low tidal field environments the slopes are steeper (more negative), representing an abundance of low EW clouds. The trend in the void slope with $\log \mathcal{T}$ shows signs of a relative discontinuity somewhere between $0.1 \lesssim \mathcal{T} \lesssim 0.3$, the range within which we hypothesize that the "true" void leaves off and the GDS begins (§5.6). The same trend appears less obviously in the plot of the intercepts (panel $b$ ). If this trend is real it would suggest that the deepest voids may have marginally more large EW clouds and marginally larger line densities than in locations closer to void edges. Note also that choosing a larger lower tide limit (upper sub-panels) is choosing smaller volumes around galaxies, and results in significantly larger line densities (panel $b$ ). The trend in the line density in GDS is consistent with $C \propto \mathcal{T}^{0.43}$. The tendency for large errors in attributed $\mathcal{T}$ to be introduced in the GDS via positional errors makes this value highly approximate.

One may conclude that there is a significant variation in the EWDF between the upper- or lower-limit tidal fields. The systematic variation of EWDF slope with $\mathcal{T}_{\text {lim }}$ is consistent with expectations of the effects 
Table 2: Fitted slopes and intercepts as a function of $\mathcal{T}$ limits

\begin{tabular}{lcccc}
\hline \hline Mean EWDF & $\mathrm{S}$ & $\sigma_{S}$ & $\mathrm{C}$ & $\sigma_{C}$ \\
$\mathcal{W} \geq 12 \mathrm{~m} \AA$ & -0.9665 & 0.0513 & 2.082 & 0.0188 \\
$\mathcal{W} \geq 32 \mathrm{~m} \AA$ & -0.8900 & 0.0664 & 1.949 & 0.0205 \\
\hline $\mathcal{T}^{U}$ & $\mathrm{~S}$ & $\sigma_{S}$ & $\mathrm{C}$ & $\sigma_{C}$ \\
0.010 & -1.4688 & 0.1954 & 1.7812 & 0.0652 \\
0.030 & -1.5277 & 0.1920 & 1.7204 & 0.0618 \\
0.100 & -1.5303 & 0.1786 & 1.7383 & 0.0545 \\
0.300 & -1.2823 & 0.1556 & 1.8233 & 0.0476 \\
1.000 & -1.1417 & 0.1318 & 1.8912 & 0.0426 \\
3.000 & -1.0267 & 0.1228 & 1.9450 & 0.0401 \\
\hline $\mathcal{T}^{L}$ & $\mathrm{~S}$ & $\sigma_{S}$ & $\mathrm{C}$ & $\sigma_{C}$ \\
0.010 & -0.6690 & 0.1495 & 2.1087 & 0.0499 \\
0.030 & -0.5974 & 0.1524 & 2.2487 & 0.0519 \\
0.100 & -0.4845 & 0.1803 & 2.5602 & 0.0648 \\
0.300 & -0.5234 & 0.2011 & 2.7865 & 0.0754 \\
1.000 & -0.5515 & 0.2533 & 2.9507 & 0.0947 \\
3.000 & -0.3349 & 0.3486 & 3.0903 & 0.1374 \\
\hline \hline
\end{tabular}

of tidal fields $(\S 3)$. The modestly slight variation in distribution functions for a range below $\mathcal{T}^{U} \approx 0.16$ indicates that the void cloud EWDF is fairly uniform over the $\approx 90 \%$ of the universe occupied by voids. The increasing concentration of clouds around galaxies, as shown by the steady steep rise in the intercepts of catalogs $\mathcal{C}\left(\mathcal{T}^{L}\right)$ as $\mathcal{T}^{L}$ increases, appears consistent with infall of clouds into the potential wells of galaxies and groups of galaxies.

\subsection{Error analysis by the variational approach}

We need to assure ourselves of the robustness of these results. In previous sections a number of possible biases, uncertainties and sources of error were noted which could not be directly evaluated except by noting the effect of different treatment of the data on the derived EWDFs. The results of these comparisons are presented below.

\subsubsection{Completeness}

Recall (§5.4) that the effect of incompleteness is to put a skew on the depth of the luminosity function probed across a range of redshifts, and hence to under-estimate $\mathcal{T}$ at the high-end of the redshift range, relative to that at low redshift. This effect is shown graphically by the dotted lines in Fig. 4, where an absolute magnitude upper-limit of -19.8 was imposed on the galaxy catalogs. In this section, the data is separated by a redshift constraint, and the EWDFs are re-derived for the low-redshift part, and compared with our standard. We make our division at $z=0.036$, at which point a apparent magnitude limit of $m_{B}=16$ means that we are seeing all but galaxies with $\mathcal{L} \leq 0.74 \mathcal{L}^{*}\left(v_{c} \simeq 145 \mathrm{~km} \mathrm{~s}^{-1}\right)$. Fig. 11 shows the 


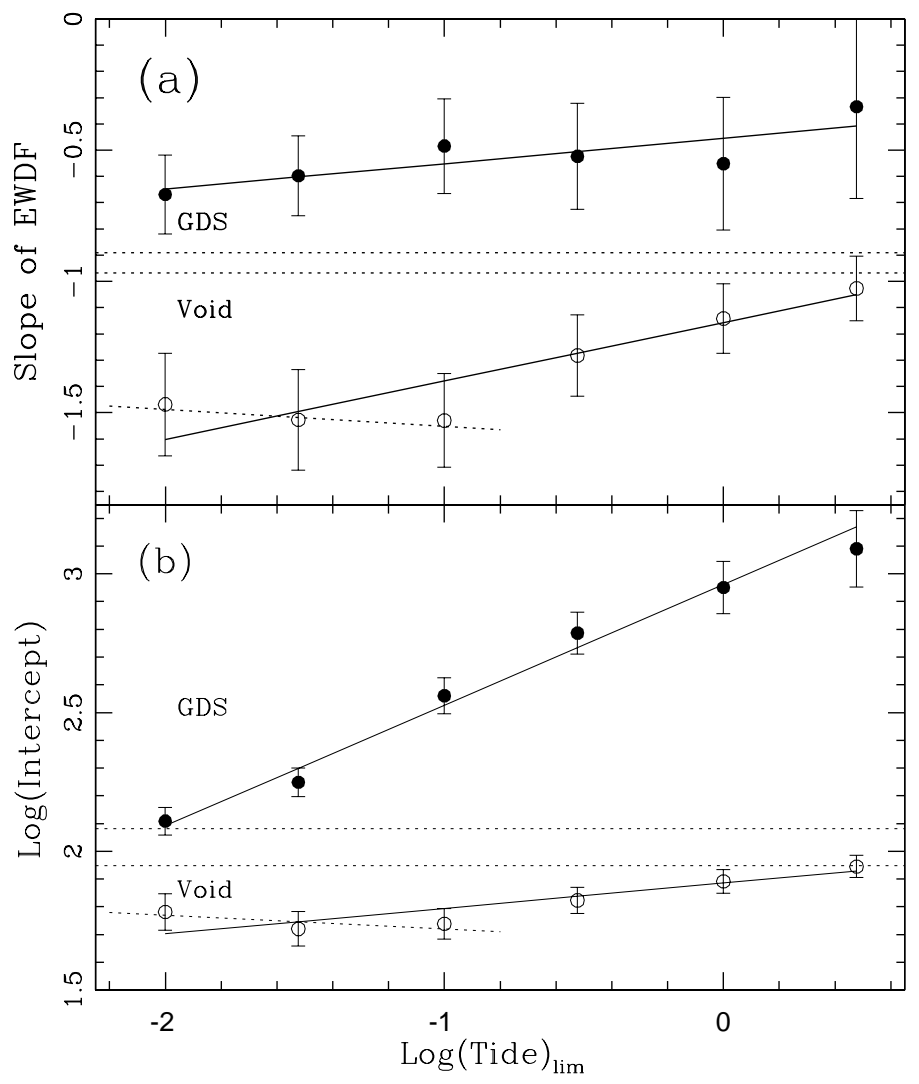

Fig. 10.- Standard model fits: The number-weighted linear fits to the slopes $S$ (panel $a$ ) and intercepts $C$ (panel $b$ ), terms defined in Eq. 45, are presented for various $\mathcal{T}$ limits of cloud catalogs (see text). The solid lines represent a linear fit to the slopes (panel $a$ ) and intercepts (panel $b$ ) as a function of the logarithm of the tide $\mathcal{T}$. In panel $a$, the upper horizontal dotted line has a value of the slope of the mean EWDF for $\mathcal{W} \gtrsim 32$ $\mathrm{m} \AA$ (the location of the inflection in the mean EWDF), and the lower, $\mathcal{W} \gtrsim 15 \mathrm{~m} \AA$. Above that (filled circles) are the EWDF slopes for catalogs $\mathcal{C}\left(\mathcal{T}^{L}\right)$ (non-void), and below the dotted line (open circles) are the slopes for $\mathcal{C}\left(\mathcal{T}^{U}\right)$ (void clouds). In panel $b$, the horizontal dotted lines represent the intercept (logarithm of $d \mathcal{N} / d z$ ) for the mean EWDF for $\mathcal{W} \geq 32 \mathrm{~m} \AA$ (lower) and for $\mathcal{W} \geq 15 \mathrm{~m} \AA$ (upper). Filled circles represent the intercepts of $\mathcal{C}\left(\mathcal{T}^{L}\right)$ EWDFs, and open circles represent the EWDF intercepts for catalogs $\mathcal{C}\left(\mathcal{T}^{U}\right)$. The short dotted lines in the void regions of panels $a$ and $b$ are linear fits to the points with $\mathcal{T}^{U} \lesssim 0.16$ indicating a possible inflection of the trend of slope and intercept with $\mathcal{T}^{U}$.

results of that analysis in comparison to that of the standard processing for catalogs based on $\mathcal{T}_{\text {lim }}=0.01$ and 0.1. Figure 12 shows the linear fits for all $\mathcal{C}(\mathcal{T})$ for $z<0.036$. The lines are the fits to data at all redshifts (Fig. 10), placed for the convenience of a visual comparison of the changes. Note that the slopes of void clouds have steepened; at $\mathcal{T}_{\text {lim }}=0.1$, the slope of EWDFs rises from $\sim-1.5$ to $\sim-1.7$, possibly a better estimate of the true EWDF slope in voids. The previous impression of an inversion of the trend of slopes for $\mathcal{T}^{U} \lesssim 0.16$ (panels $a$ and $b$, short dotted lines from Fig. 10) is now strengthened in significance (dashed lines). Though the number statistics are now weaker, it appears that we may have gained in some respects by discarding higher-redshift data, for the feature at $\mathcal{T} \simeq 0.16$ in Fig. 12 which was somewhat weak in the standard processing using all the data now appears stronger with the lower-redshift data. This 
could be expected because fewer clouds from higher tidal field environments may contaminate the low tide catalogs when higher redshift data are culled.

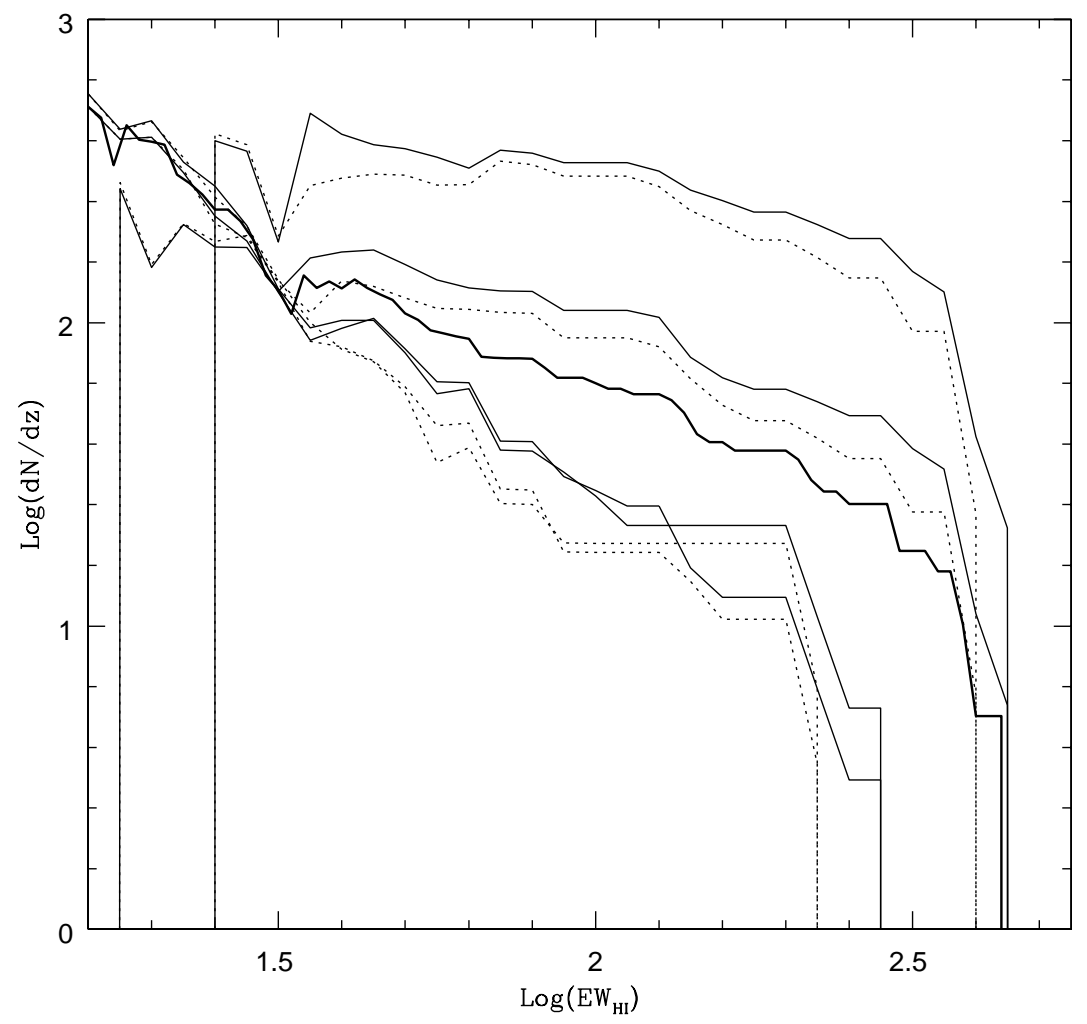

Fig. 11. - Completeness test: The equivalent width distribution functions ( $\mathcal{T}_{\text {lim }}=0.01$ and 0.1 , with (solid) and without (dotted), the redshift interval $z \geq 0.036$. The median EWDF is plotted (heavy solid line) for reference. The $\mathcal{T}^{U}$ (void) EWDFs are evidently somewhat steeper with the redshift cutoff; for $\mathcal{T}^{U}=0.1$, the slope steepened from -1.56 to -1.70 . The $\mathcal{T}^{L}$ EWDFs (upper pairs of lines) show a drop with the rejection of higher redshift data. This may be consistent with evolution effects.

\subsubsection{Effect of early-type galaxies}

Recall (§5.1) that in attributing mass to galaxies the TF relation was used to transform the absolute magnitudes into galaxy masses. Early-type galaxies do not follow the TF relation, however, they do follow a quite similar relation, basically $\mathcal{L} \propto \sigma^{\sim 4}$. This is steeper than the TF relation, but the main effect, for our purposes, is that the mass-luminosity ratio is larger for early-type galaxies. This can be checked by alternatively using, or not using, an altered attributed mass for those galaxies listed in the CfA catalog as having negative de Vaucouleurs T-Types (those are generally lenticular or elliptical galaxies). The alteration involves multiplying the normally attributed mass by a factor of 3.0 when the T-Type is less than or equal to zero. Since ellipticals are more often found in clusters, we might expect that this would attribute more masses to large groups, or clusters of galaxies, inflating the $\mathcal{T}^{L}$ contours somewhat, which would reduce the EWDF for the GDS at a given $\mathcal{T}^{L}$. There should be relatively little effect on the void EWDFs since clouds are thought to avoid high density regions (Morris et al. 1991, 1993) where early-type galaxies are 


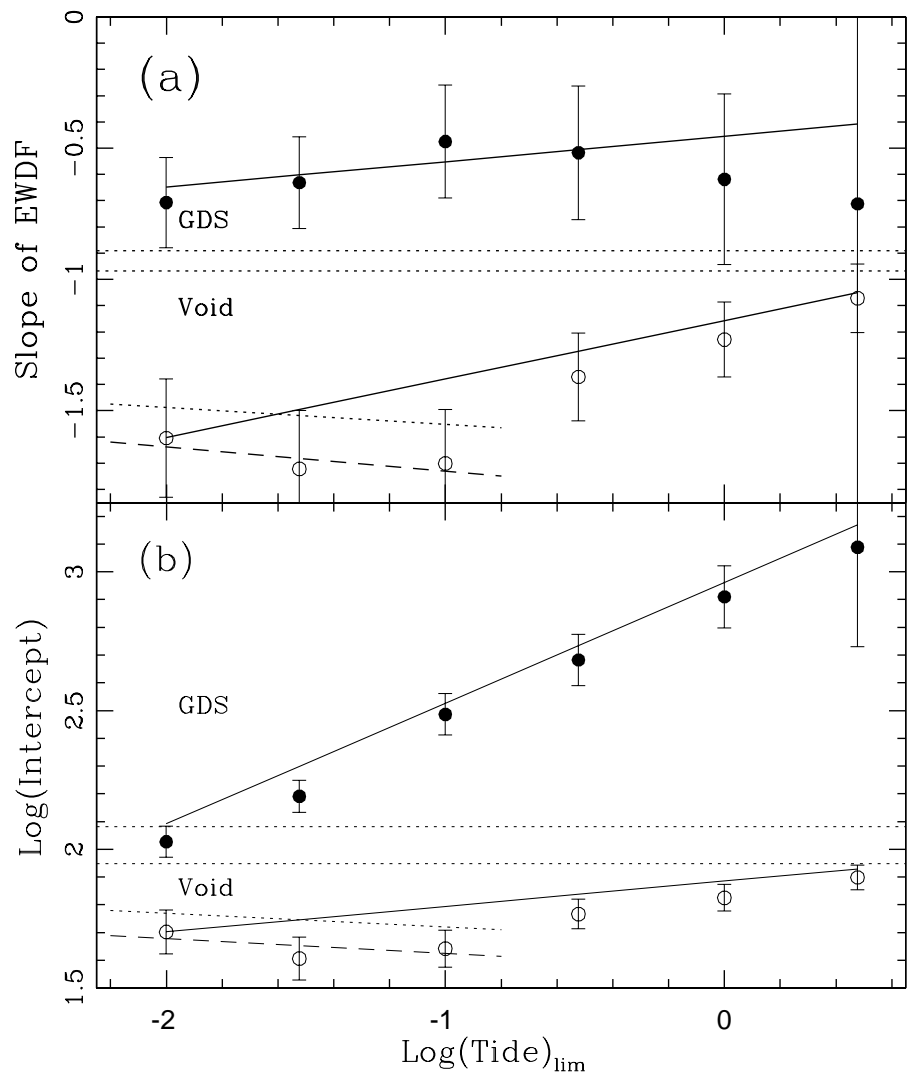

Fig. 12.- Completeness test: Same as Fig. 13, except that slopes and intercepts are fit to catalogs $\mathcal{C}$ constrained by $z \leq 0.036$, and the solid lines are the previous fits found for catalogs with no redshift constraint for comparison. What appeared as a marginal trend for increase in slope for GDS has now disappeared, though the intercept relation remains relatively accurate (top sub-panels of panels $a$ and $b$, respectively). Note that what was previously a marginal tendency for slopes and intercepts of void clouds with $\mathcal{T}_{\text {lim }} \lesssim 0.16$ (short, dotted lines) to have a trend contrary to the general trend suggested by the linear fit, is now more significant (short, dashed lines). This provides evidence for a relatively sharp change of character of cloud distributions near $\mathcal{T}_{\text {lim }} \simeq 0.16$ with the truncated isothermal model.

concentrated.

As can be seen in Fig. 13, there is slight reduction in the line density of the DFs. The reason for this is probably that the added mass simply increases the volume of space at a given tidal field limit, which then has a slightly lower EWDF, as noted in Fig. $10 b$.

\subsubsection{Effects of halo type}

The NFW halo is significantly less massive than the truncated isothermal halo ( $\$ 5.1$, and Fig. 3), and therefore when halos of galaxies are interpreted as NFW, the main effect is that the tidal fields will be lower by a factor of $\sim 5$. Thus we can expect that the intercepts and slopes will be shifted to lower $\log \mathcal{T}$ by a factor of $\approx 0.7$. These results are presented in the form of fitted slopes and intercepts of number-weighted 


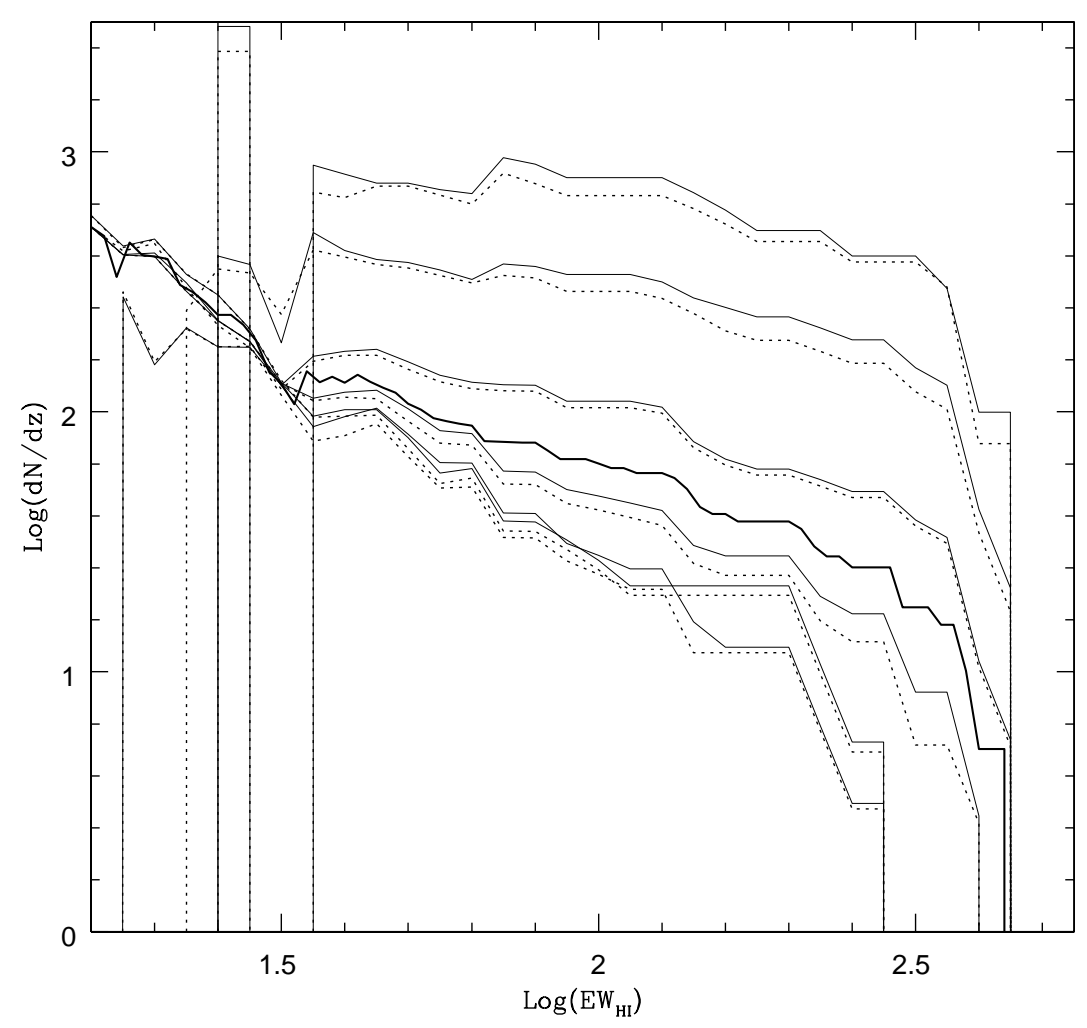

Fig. 13. - The T-Type test: The equivalent width distribution functions with (dotted lines), and without (solid), the T-Type corrections for early-type galaxies, which makes the attributed galaxy mass 3 times greater. Tide limits are $\mathcal{T}_{\text {lim }}=0.01,0.1,1.0$. The tendency is for EWDFs based on tidal field lower limits to be more influenced in high tidal field, than low tidal field environments, though only marginally so.

linear fits in Fig. 14.

It is difficult to judge from Figs. 10 and 14 alone which halo is most likely to be more correct. However, we saw signs in Fig. 8. on the basis of most probable b-values, that the transition from void to GDS clouds occurs in the range $0.1 \lesssim \mathcal{T} \lesssim 0.3 . \mathrm{A} \log (\mathcal{T})$ offset of -0.7 would place this transition at $\mathcal{T} \approx 0.03$ $(\log \mathcal{T} \simeq-1.5)$. While neither of these tides could not reasonably be responsible for truncating the clouds in the transition zone, at $z=1$, a lookback time of $57 \%$ of the age of the universe, the expected tidal field is $\sim 8$ times greater, which according to Eq. 19, constrains stable clouds to have densities greater than $\sim 2.5$, and $0.48 \rho_{\text {crit }}$ for isothermal and NFW halos, respectively. However, it does not seem reasonable to expect that the GDS and void clouds could be distinguished at a level as low as $0.5 \rho_{\text {crit }}$ level; clouds of such low density could not reasonably be expected to be detected with the current technology. Such a cloud in the Hubble flow would produce a column of only $10^{12} \mathrm{~cm}^{-2}(\mathcal{W} \lesssim 4 \mathrm{~m} \AA)$ over a velocity range of $\sim 220 \mathrm{~km} \mathrm{~s}^{-1}$. Hence, it appears that gas at a density of $\sim 2-5 \Omega_{b} \rho_{\text {crit }}$ is being stripped at the void-GDS boundary, where the tidal fields by themselves, even in the last few Gyr, could not reasonably be expected to be effective, unless galaxy halos are significantly more massive than even the isothermal model used here. 


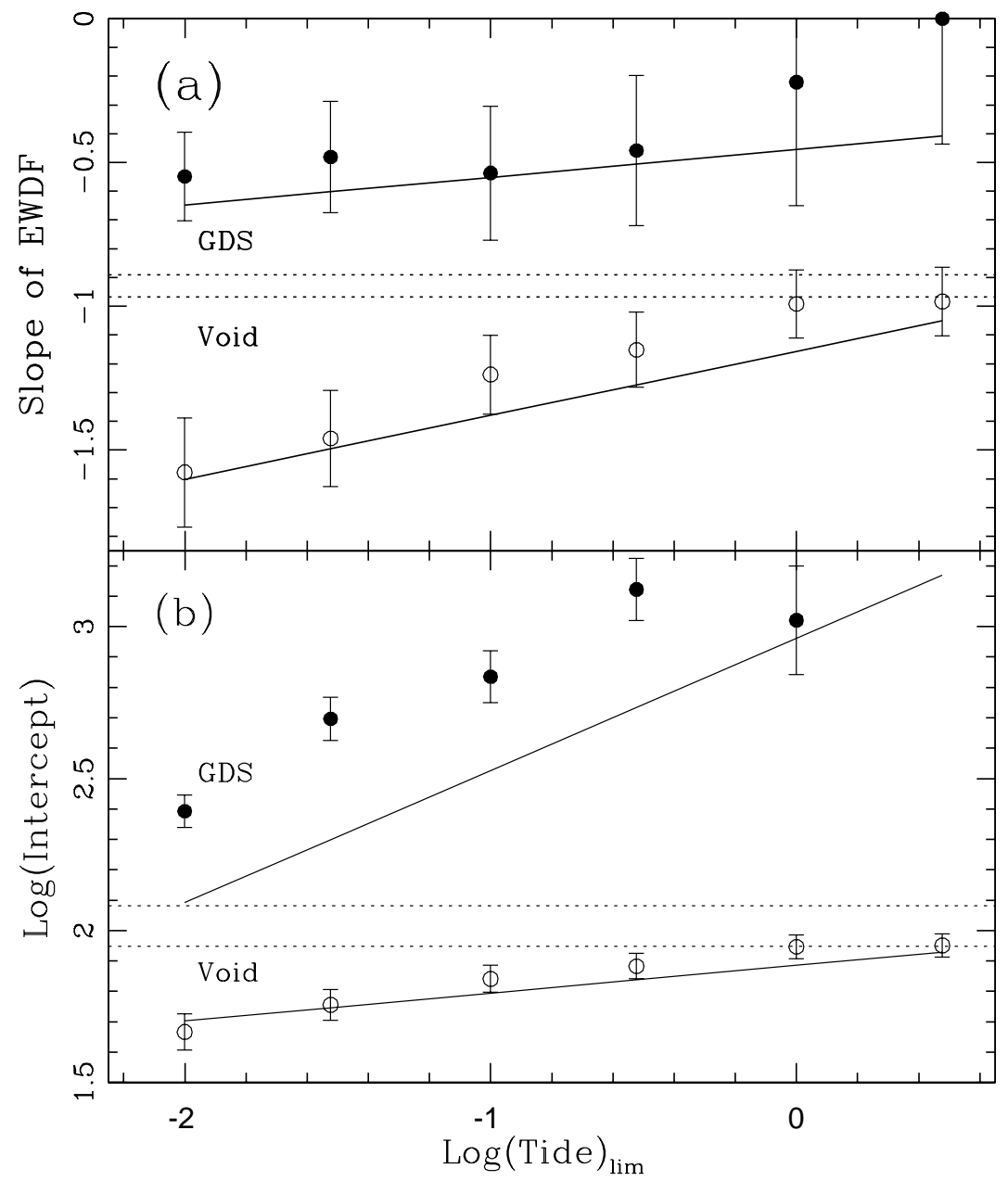

Fig. 14.- Halo-type test: Same as Fig. 10, except that slopes and intercepts are fit to catalogs $\mathcal{C}$ with tides generated by halos interpreted as NFW. The solid lines are the previous fits found for catalogs with isothermal halos. To first order the points are displaced by $\sim 0.7$ to the left (in $\log \mathcal{T}$ ) relative to the points in Fig. 10 for the isothermal halo due to the smaller NFW halo mass.

\section{Discussion}

Numerous 3-D cosmological simulations have suggested that voids have a low density, and would present very few absorbers above present-day obsesrving limits. However, careful separation of clouds into catalogs according to the ambient tidal field has resulted in the discovery of a void cloud population that is by no means insignificant. The line density of void clouds reaches a value $d \mathcal{N} / d z \approx 500$ for $\mathcal{W} \geq 10^{1.2} \approx 15.8 \mathrm{~m} \AA$. An order of magnitude calculation may convey the meaning of this. Equation 4 shows that at $z=0$, and $r_{p}=50 \mathrm{kpc}$, the comoving density $n \simeq 16 \mathrm{Mpc}^{-3}$, and the mean free path between clouds $\widehat{l} \simeq 8 \mathrm{Mpc}$, or 2.4 $\AA$ A An average cloud mass of $10^{9} \mathrm{M}_{\odot}$ (a reasonable mass for a just-detectable truncated isothermal halo of circular velocity $v_{c} \simeq 11 \mathrm{~km} \mathrm{~s}^{-1}$ ) implies $\Omega_{m}=0.1$ in voids. However, an inter-cloud medium of unknown density must exist which may add substantially to the density in voids.

Supporting the contention that these are actually void clouds is the clear distinction between the slopes of 
EWDFs based on the complementary catalogs $\mathcal{C}$ distinguished by limiting tidal fields $\mathcal{T}_{\text {lim }}$, and the difference in the most probable $b$-values of their clouds. Under the truncated isothermal halo model, the transition from void to GDS environments occurs at $\log \mathcal{T} \approx 0.8 \pm 0.2$, or $\mathcal{T} \approx 0.16$. Not only do the slopes and intercepts of the void EWDFs undergo a strong change (Figs. 10, 12), but the differential histogram of cloud $b$-values also shows the transition. At this tidal field limit, $~ 90 \%$ of the universe is void, and $10 \%$ is GDS.

That the change in cloud character noted above can be associated with a certain tidal field limit under a given assumed halo type supports the contention that tidal fields are a relevant quantity, associating cloud stability and cloud characteristics with void and GDS environments. It further supports the contention that clouds are self-gravitating to some extent, for the variation of EWDF with tidal field is consistent with the predicted effects on such clouds. In the case of void clouds, the lack of strong tides allows a distribution of clouds which is unconstrained to the current detection limits, allowing small, delicate structures to exist. However, their true nature cannot be known without successful modeling of them in cosmological simulations. In the case of GDS clouds, the tidal truncation which galaxies and groups of galaxies impose on clouds appears to have resulted in a paucity of low-EW clouds, and a general flattening of the EWDFs. On the other hand, if GDS environment is well-characterized by the filamentary structures seen in 3-D hydro simulations, and have a relatively high density and temperature, it is possible that much of the observed truncation is by ram pressure and shear forces as clouds fall into the GDS from voids. This would solve the problem (noted in §6.2.3) of tidal field levels at the location of the apparent transition between void and GDS being too low with either halo model to easily reconcile with the probable density of gas being stripped from clouds moving from void to the GDS. Both effects have a common source in the gravitational potential of the mass concentration, and a similar result in the stripping of low-density gas, so that even if ram-pressure stripping is a major factor in the difference between void and GDS clouds, the tidal field is still an effective and valid way to separate void and GDS clouds.

There is a hint of structure in voids - deep voids appear to have marginally larger line densities, and shallower EWDF slopes; a hint of more massive clouds there. This may suggest some degree of hierarchical clustering has taken place.

In comparing ISOT and NFW models, the most obvious difference is that in mass (NFW is a factor of 4 to 5 smaller). The NFW halo has a weaker ability to tidally truncate clouds, and to maintain the filling factor of 0.1 for GDS, one requires that $\mathcal{T}_{\text {lim }} \simeq 0.03$ has in the past tidally truncated the GDS clouds (see $\S 6.2 .4)$. But this may be too small to reasonably have tidally truncated those clouds. In addition, the scaling of the absorption radius with luminosity in the $B$-, and $K$-bands (Chen et al. 2001) agree nicely with the scaling relations of ISOT (Eq. 29), but do not fit well with the NFW halo (Eq. 40 - see $\S 55.1 .1,5.1 .2$ ).

Though I consider these general findings to be secure, many improvements could be made in the quality and quantity of data. For instance, the depth and quality of the data in the CfA redshift catalog leaves much to be desired. However, when the galaxy catalogs from the Sloan Digital Sky Survey become available, much of these problems will be solved; both deeper $\left(m_{g^{*}}=17.65 \mathrm{mag}\right)$, more consistent photometry, and generally superior redshifts will be available, so that tidal fields may be calculated to greater accuracy.

The quantity of absorption systems, and the sensitivity of the observations also leaves something to be desired. However, there will soon be published results of Space Telescope Imaging Spectrograph (STIS) observations of $\sim 120$ new absorbers (M. Shull, priv. comm.), more than doubling the current number of absorbers. Somewhat farther in the future, the Cosmic Origins Spectrograph (COS) will provide significantly deeper spectrography of low redshift AGN, and should greatly increase the number of $\mathrm{HI}$ absorbers available for study. 
While this analysis has displayed the nature of the distinction between void, and GDS clouds, we know little about the clouds themselves. The EWDF is just a sampling of the clouds weighted by cloud crosssection at a given EW. We do not know their mass, their cross-section, or their density. We have a tentative cloud model - clouds of primordial gas whose expansion is restrained by dark matter halos - but do not know in any detail what parent population has given rise to these absorbers, nor what contribution they make to $\Omega_{m}$. Though analytical models may help to clarify what the current state of clouds might be, the accurate implementation of the cloud hypothesis adopted here requires a hydrodynamical study beginning at the point of reionization in order to assess the current extent and dynamical characteristics of void clouds under a variety of halo models. These results could be used to substantiate, or invalidate, those models. If the former, it would help determine the number and mass-distribution of clouds which are consistent with the observed void EWDF, and thus estimate their contribution to $\Omega_{m}$. This task remains for a paper now in preparation.

\section{Conclusions}

The large line density of clouds in voids, where simulations have predicted very few clouds at EWs accessible to even STIS observations (R. Davé, priv. comm. 2001), appears to require massive dark halos to restrain the dispersal of gas needed to produce detectable absorbers. We have seen that such halos are consistent with the general predictions of hierarchical structure formation if small halos did not generally form Population III stars. We further find that the increase in line density of clouds with increasing tidal fields in GDS are consistent with continuing infall of clouds from void regions into the GDS.

We make the following specific conclusions.

1. Tidal fields are a convenient and effective parameter with which to separate clouds into the complementary environments; "void", and "galaxy dominated" spaces. However, it is possible that the tidal field stripping of clouds is significantly supplemented in GDS by ram-pressure stripping by the diffuse, ambient dissipated gas populating filamentary structures which appear in 3-D cosmological simulations.

2. The EWDF is significantly steeper in voids than the median EWDF, suggesting that the void cloud population has most of its mass at smaller scales.

3. Most probable void cloud Doppler parameters are characteristically half that of GDS clouds.

4. The line density in GDS is of order more than 10 times larger for $\mathcal{W} \geq 63 \mathrm{~m} \AA$, but for $\mathcal{W} \lesssim 35 \mathrm{~m} \AA$, void clouds dominate the mean EWDF.

5. The void cloud EWDF is essentially constant over $\sim 90 \%$ of the universe, while the GDS EWDFs have line densities that increase dramatically with increases in $\mathcal{T}^{L}$.

We have analyzed the void-cloud population and found significant differences between that and the picture provided by 3-D hydrodynamic simulations. It is speculated that the lack of resolving power in the simulations may be partially responsible for this difference. However, it appears that even so, the sheer number density of clouds which must be in voids is startling, and may presage a shift in thought about Ly $\alpha$ clouds.

I am very grateful to Hy Spinrad, who provided support for this research, and helpful suggestions. I am also grateful for the many comments and helpful suggestions from Christopher F. McKee. I thank Steve 
Penton and J. Michael Shull for their help with the sensitivity functions and cloud identification issues. Finally, I wish to thank the anonymous referee for many helpful suggestions and comments.

\section{APPENDIX}

\section{TRUSTWORTHINESS OF THE CfA REDSHIFT CATALOG}

A lot will depend on the reliability of the redshifts and magnitudes in the assembled catalogs. Since the CfA galaxy catalog is a compilation of data from many sources, its tabulated magnitudes are somewhat inhomogeneous. To check the validity of these magnitudes, the galaxy positions are used to query the Automated Plate Scanner Facility at the University of Minnesota (e.g., Pennington et al. (1993) $)^{5}$. The APS is based on digitized Palomar Observatory Sky Survey (DPOSS) plates. Not all galaxies were "found" by the APS. Reasons were often that the relevant plate was not mounted. Sometimes the galaxy was simply not found, though STScI digitized sky survey images ${ }^{6}$ clearly showed their presence (often these were edgeon spirals). In a very few cases, nothing was found. Roughly half of the CfA galaxies were matched by an APS galaxy. The apparent magnitudes are converted to absolute magnitudes, corrected for galactic extinction (Schlegel et al. 1998), and inclination (Tully et al. 1998). A scatter plot makes it clear that some of the magnitudes were anomalous. However, the overwhelming majority of galaxies fit the relation, $M_{A P S}=M_{C f A}+(0.65 \pm 0.5) \mathrm{mag}$, with a slope of 1.004

The stated error of the APS magnitudes for integrated isophotal galaxy magnitudes (Pennington et al. 1993 ) was estimated to be $\sigma_{A P S} \simeq 0.3$ to $0.5 \mathrm{mag}$, but a more recent estimate is $0.3 \mathrm{mag}$ (R. Humphreys, priv. comm., 2001), reflecting the added sophistication of their processing. The observed dispersion is 0.5 mag in the APS-CfA plot, which is also the dispersions of APS and CfA magnitudes combined in quadrature. Thus, we estimate $\sigma_{C f A} \simeq 0.4 \mathrm{mag}$.

The 0.65 mag offset indicates that the APS magnitudes are fainter. We shall see that much of this offset can be understood in terms of the different spectral band in which the magnitudes are measured. The CfA magnitudes are generally B-band magnitudes with $\lambda_{\text {eff }} \simeq 4200-4400 \AA$. The APS magnitudes are POSS O-band with $\lambda_{\text {eff }} \simeq 4000 \AA$. A compilation of published studies of luminosity functions (LF) in a range of spectral bands (Blanton et al. 2001; Cole et al. 2001; Mobasher, Sharples, \& Ellis 1993; Tully 1988; Tully \& Pierce 2000) show how the defining parameters of the fit vary with the spectral band, revealing a tight relation between $\phi^{*}$, and $M^{*}$ (Fig. A1a, c) as a function of spectral band. Let us analyze the CfA versus the APS magnitudes in the light of this figure. Note first that as one chooses bluer bands, the galaxy luminosity is smaller at the "knee" of the luminosity function, and the normalization $\phi^{*}$ is larger.

It is the Tully-Fisher relation (Tully et al. 1998) which allows us to use the LF data to get our answer. TF can be expressed,

$$
M_{\text {band }}=M_{\text {band }}^{*}-2.5\left[\beta_{\text {band }}\left\{\log \left(2 v_{c}\right)-\log \left(2 v_{0}\right)\right\}\right] .
$$

Applying this once each to $O$ and $B$ magnitudes and subtracting them, we have the transform from $O$ to $B$ for a galaxy of circular velocity $v_{c}$,

$$
B=O-\left(O^{*}-B^{*}\right)+2.5\left\{\beta_{0}-\beta_{B}\right\} \log \left(\frac{v_{c}}{v_{0}}\right)
$$

\footnotetext{
${ }^{5}$ The APS databases are supported by the National Aeronautics and Space Administration and the University of Minnesota. The APS databases can be accessed at http://aps.umn.edu/.

${ }^{6}$ The Digitized Sky Surveys were produced at the Space Telescope Science Institute under the U. S. Government grant NAG $\mathrm{W}-2166$, and may be accessed at http ://archive.stsci.edu/cgi-bin/dss form
} 


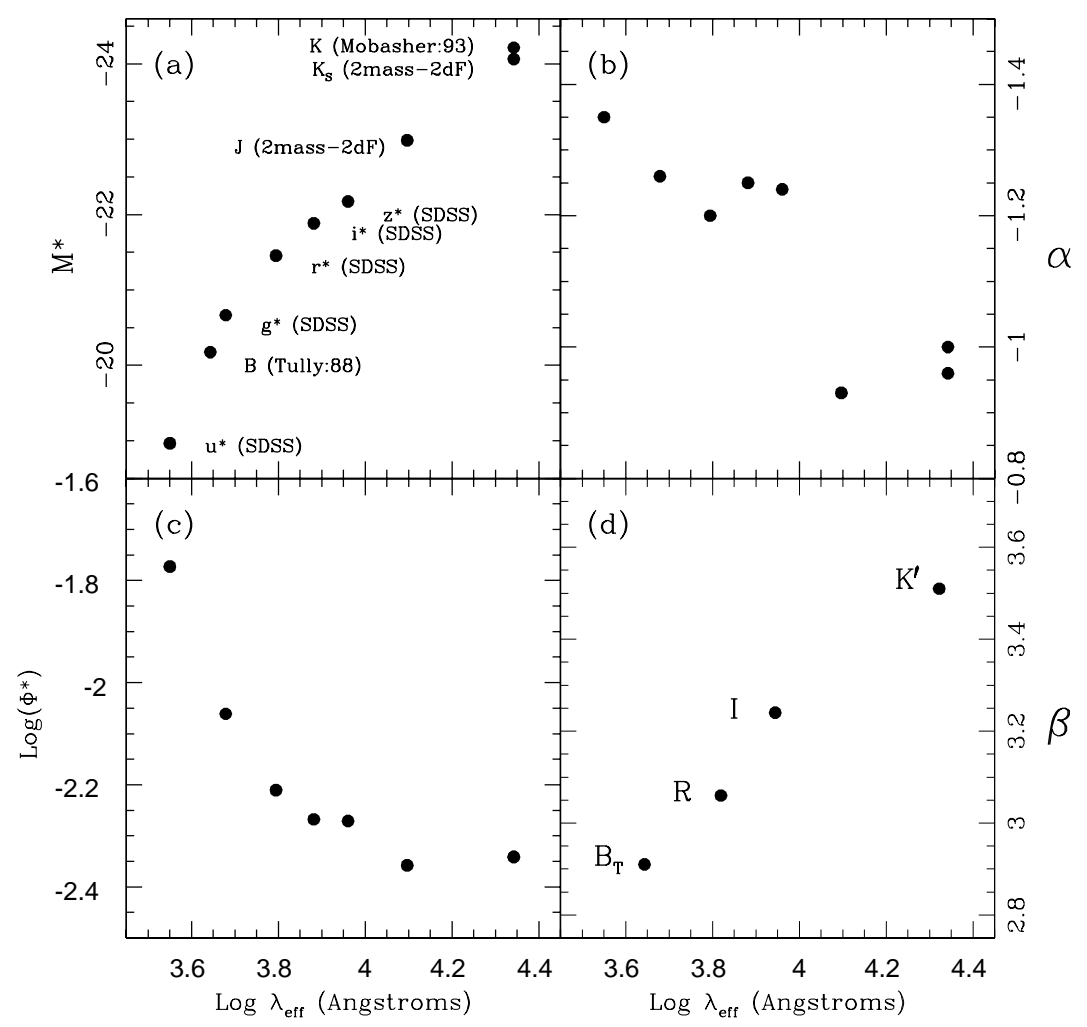

Fig. 1.- Panels (a) through (c) show luminosity function parameters. Panel (a) shows $M^{*}$ as a function of various spectral bands using $h=0.75$ (Blanton et al. 2001; Cole et al. 2001; Mobasher et al. 1993; Tully 1988). Panel (b) shows the faint-end slope $\alpha$ as a function of $\lambda_{\text {eff }}$. Panel (c) shows the log of the normalization $\Phi^{*}$ with $h=0.75$. Panel (d) shows the slope $\beta$ (see Eq. 26, §5.1) of the Tully-Fisher relation as a function of spectral band (Tully \& Pierce 2000).

where $\beta_{\text {band }}$ is the Tully-Fisher slope (see Fig. A1d), and $v_{0}$ is a fiducial velocity of $158 \mathrm{~km} \mathrm{~s}^{-1}$. A linear fit of the $B_{T}, R$, and $I$ bands (Fig. A1d) implies $\beta_{0} \simeq 2.83$, and $\beta_{B} \approx 2.87$ to 2.89. $M^{*}$ and $\phi^{*}$ change relatively fast in these bands $\left(M_{O}^{*}=-19.0 \mathrm{mag}, \phi_{O}^{*} \simeq 0.029 \mathrm{~h}^{3} \mathrm{Mpc}^{-3}\right.$ and $M_{B}^{*}=19.3-19.7 \mathrm{mag}$, $\phi_{B}^{*} \simeq 0.026-0.023 \mathrm{~h}^{3} \mathrm{Mpc}^{-3}$ ) for the $4200 \AA$ and $4400 \AA$ versions of $B$, respectively.

Applying these values of $M^{*}$ to Eq. 1 shows a shift of +0.4 to 0.7 magnitudes for a galaxy with $v_{c}=158 \mathrm{~km} \mathrm{~s}^{-1}$ going from $B$ to $O$ band magnitudes; $O$ is "fainter". For galaxies with $v_{c}$ smaller than $158 \mathrm{~km} \mathrm{~s}^{-1}$, there is only a slight skew in the sense that for small galaxies, the shift in attributed absolute magnitude is slightly larger.

One can conclude that the CfA magnitudes are generally consistent, and trustworthy within the constraints established above.

\section{REFERENCES}

Abel, T., Anninos, P., Norman, M. L., \& Zhang, Y. 1998, ApJ, 508, 518 
Avila-Reese, V., Firmani, C., Klypin, A., \& Kravtsov, A. V. 1999, MNRAS, 310, 527

Bahcall, J. N., Bergeron, J., Boksenberg, A., Hartig, G. F., Jannuzi, B. T., Kirhakos, S., Sargent, W. L. W., Savage, B. D., Schneider, D. P., Turnshek, D. A., Weymann, R. J., \& Wolfe, A. M. 1993, ApJS, 87, 1

-. 1996, ApJ, 457, 19

Bahcall, J. N., Jannuzi, B. T., Schneider, D. P., Hartig, G. F., Bohlin, R., \& Junkkarinen, V. 1991, ApJ, $377, \mathrm{~L} 5$

Bahcall, J. N., Jannuzi, B. T., Schneider, D. P., Hartig, G. F., \& Green, R. F. 1992, ApJ, 397, 68

Bahcall, N. A. \& Cen, R. 1993, ApJ, 407, L49

Bahcall, N. A., Lubin, L. M., \& Dorman, V. 1995, ApJ, 447, L81

Barkana, R. \& Loeb, A. 1999, ApJ, 523, 54

Bechtold, J. 1994, ApJS, 91, 1

Blanton, M. R., Dalcanton, J., Eisenstein, D., Loveday, J., Strauss, M. A., SubbaRao, M., Weinberg, D. H., Anderson, J. E., Annis, J., Bahcall, N. A., Bernardi, M., Brinkmann, J., Brunner, R. J., Burles, S., Carey, L., Castander, F. J., Connolly, A. J., Csabai, I. ., Doi, M., Finkbeiner, D., Friedman, S., Frieman, J. A., Fukugita, M., Gunn, J. E., Hennessy, G. S., Hindsley, R. B., Hogg, D. W., Ichikawa, T., Ivezić, Ž., Kent, S., Knapp, G. R., Lamb, D. Q., Leger, R. F., Long, D. C., Lupton, R. H., McKay, T. A., Meiksin, A., Merelli, A., Munn, J. A., Narayanan, V., Newcomb, M., Nichol, R. C., Okamura, S., Owen, R., Pier, J. R., Pope, A., Postman, M., Quinn, T., Rockosi, C. M., Schlegel, D. J., Schneider, D. P., Shimasaku, K., Siegmund, W. A., Smee, S., Snir, Y., Stoughton, C., Stubbs, C., Szalay, A. S., Szokoly, G. P., Thakar, A. R., Tremonti, C., Tucker, . 2001, AJ, 121, 2358

Blitz, L., Spergel, D. N., Teuben, P. J., Hartmann, D., \& Burton, W. B. 1999, ApJ, 514, 818

Braun, R. \& Burton, W. B. 2000, A\&A, 354, 853

Bromm, V., Coppi, P. S., \& Larson, R. B. 1999, ApJ, 527, L5

Burkert, A. 2000, ApJ, 534, L143

Carraro, G., Ng, Y. K., \& Portinari, L. 1998, MNRAS, 296, 1045

Cen, R. \& Ostriker, J. P. 1999, ApJ, 514, 1

Chen, H., Lanzetta, K. M., Webb, J. K., \& Barcons, X. 1998, ApJ, 498, 77

-. 2001, ApJ, 559, 654

Cole, S., Norberg, P., Baugh, C. M., Frenk, C. S., Bland-Hawthorn, J., Bridges, T., Cannon, R., Colless, M., Collins, C., Couch, W., Cross, N., Dalton, G., De Propris, R., Driver, S. P., Efstathiou, G., Ellis, R. S., Glazebrook, K., Jackson, C., Lahav, O., Lewis, I., Lumsden, S., Maddox, S., Madgwick, D., Peacock, J. A., Peterson, B. A., Sutherland, W., \& Taylor, K. 2001, MNRAS, 326, 255

Davé, R., Hernquist, L., Katz, N., \& Weinberg, D. H. 1999, ApJ, 511, 521

Edvardsson, B., Andersen, J., Gustafsson, B., Lambert, D. L., Nissen, P. E., \& Tomkin, J. 1993, A\&A, 275, 101 
Einasto, J., Saar, E., Einasto, M., Freudling, W., \& Gramann, M. 1994, ApJ, 429, 465

El-Ad, H. \& Piran, T. 1997, ApJ, 491, 421

Firmani, C., D’Onghia, E., Chincarini, G., Hernández, X., \& Avila-Reese, V. 2001, MNRAS, 321, 713

Grebel, E. K., Braun, R., \& Burton, W. B. 2000, in American Astronomical Society Meeting, Vol. 196, 2809

Grogin, N. A., Geller, M. J., \& Huchra, J. P. 1998, ApJS, 119, 277

Haynes, M. P. 2001, in Gas and Galaxy Evolution, ASP Conference Proceedings, Vol. 240. Edited by John E. Hibbard, Michael Rupen, and Jacqueline H. van Gorkom. San Francisco: Astronomical Society of the Pacific, ISBN: 1-58381-077-3, 2001, p. 257, 257

Hernquist, L., Katz, N., Weinberg, D. H., \& Jordi, M. 1996, ApJ, 457, L51

Huchra, J. P., Geller, M. J., \& Corwin, H. G. 1995, ApJS, 99, 391

Huchra, J. P., Geller, M. J., de Lapparent, V., \& Corwin, H. G. 1990, ApJS, 72, 433

Huchra, J. P., Vogeley, M. S., \& Geller, M. J. 1999, ApJS, 121, 287

Kauffmann, G., White, S. D. M., \& Guiderdoni, B. 1993, MNRAS, 264, 201

Kim, T., Hu, E. M., Cowie, L. L., \& Songaila, A. 1997, AJ, 114, 1

Klypin, A., Kravtsov, A. V., Valenzuela, O., \& Prada, F. 1999, ApJ, 522, 82

Lanzetta, K. M., Bowen, D. B., Tytler, D., \& Webb, J. K. 1995, ApJ, 442, 538

Lu, L., Wolfe, A. M., \& Turnshek, D. A. 1991, ApJ, 367, 19

Maciel, W. J. \& Rocha-Pinto, H. J. 1998, MNRAS, 299, 889

Marzke, R. O., Huchra, J. P., \& Geller, M. J. 1996, AJ, 112, 1803

Mobasher, B., Sharples, R. M., \& Ellis, R. S. 1993, MNRAS, 263, 560

Moore, B., Ghigna, S., Governato, F., Lake, G., Quinn, T., Stadel, J., \& Tozzi, P. 1999a, ApJ, 524, L19

Moore, B., Quinn, T., Governato, F., Stadel, J., \& Lake, G. 1999b, MNRAS, 310, 1147

Morris, S. L., Weymann, R. J., Anderson, S. F., Hewett, P. C., Francis, P. J., Foltz, C. B., Chaffee, F. H., \& MacAlpine, G. M. 1991, AJ, 102, 1627

Morris, S. L., Weymann, R. J., Dressler, A., McCarthy, P. J., Smith, B. A., Terrile, R. J., Giovanelli, R., \& Irwin, M. 1993, ApJ, 419, 524

Mücket, J. P., Petitjean, P., Kates, R. E., \& Riediger, R. 1996, A\&A, 308, 17

Nakamura, F. \& Umemura, M. 2001, ApJ, 548, 19

Navarro, J. F., Frenk, C. S., \& White, S. D. M. 1996, ApJ, 462, 563

Navarro, J. F., Frenk, C. S., \& White, S. D. M. 1997, ApJ, 490, 493 
Pennington, R. L., Humphreys, R. M., Odewahn, S. C., Zumach, W., \& Thurmes, P. M. 1993, PASP, 105, 521

Penton, S. V., Stocke, J. T., \& Shull, J. M. 2000, ApJS, 130, 121 (PSSI)

Penton, S. V., Shull, J. M., \& Stocke, J. T. 2000a, ApJ, 544, 150 (PSSII)

Riediger, R., Petitjean, P., \& Mucket, J. P. 1998, A\&A, 329, 30

Sargent, W. L. W., Young, P. J., Boksenberg, A., \& Tytler, D. 1980, ApJS, 42, 41

Schlegel, D., Finkbeiner, D., \& Davis, M. 1998, ApJ, 500, 525

Scott, D. and Silk, J. and Kolb, E. W. and Turner, M. S. 2000, in Allen's Astrophysical Quantities, ed., Cox, N., Springer-Verlag, New York, Berlin, Heidelberg

Shull, J. M., Roberts, D., Giroux, M. L., Penton, S. V., \& Fardal, M. A. 1999, AJ, 118, 1450

Theuns, T., Leonard, A., \& Efstathiou, G. 1998, MNRAS, 297, L49

Tripp, T. M., Lu, L., \& Savage, B. D. 1998, ApJ, 508, 200

Tully, R. B. 1988, AJ, 96, 73

Tully, R. B. \& Fisher, J. R. 1977, A\&A, 54, 661

Tully, R. B. \& Pierce, M. J. 2000, ApJ, 533, 744

Tully, R. B., Pierce, M. J., Huang, J., Saunders, W., Verheijen, M. A. W., \& Witchalls, P. L. 1998, AJ, 115, 2264

Tully, R. B. \& Shaya, E. J. 1984, ApJ, 281, 31

Wakker, B. P., Howk, J. C., Savage, B. D., van Woerden, H., Tufte, S. L., Schwarz, U. J., Benjamin, R., Reynolds, R. J., Peletier, R. F., \& Kalberla, P. M. W. 1999, Nature, 402, 388

Weymann, R. J., Jannuzi, B. T., Lu, L., Bahcall, J. N., Bergeron, J., Boksenberg, A., Hartig, G. F., Kirhakos, S., Sargent, W. L. W., Savage, B. D., Schneider, D. P., Turnshek, D. A., \& Wolfe, A. M. 1998, ApJ, 506,1

Wu, X. \& Xue, Y. 2000, ApJ, 542, 578

Zaritsky, D. 1995, ApJ, 448, L17

Zaritsky, D. \& Rix, H. 1997, ApJ, 477, 118

Zaritsky, D., Smith, R., Frenk, C., \& White, S. D. M. 1997, ApJ, 478, 39

Zaritsky, D. \& White, S. D. M. 1994, ApJ, 435, 599

Zaroubi, S. \& Hoffman, Y. 1993, ApJ, 416, 410 